\title{
Delineate clonal dynamics of ovarian cancer initiating cells during pre-malignant progression using a mouse genetic mosaic system
}

Jianhao Zeng ${ }^{a}$, Eli Casarez ${ }^{a}$, Ying Jiang ${ }^{a}$, Astrid Catalina Alvarez-Yela ${ }^{\mathrm{b}}$, Brianna E. Kelly ${ }^{\mathrm{a}}$, Eugene Ke ${ }^{\mathrm{a}}$, Taylor M.Jenkins ${ }^{c}$, Kristen A. Atkins ${ }^{c, d}$, Kevin A. Janes ${ }^{b, d}$, Jill K. Slack-Davis ${ }^{a, d^{*}}$, Hui Zong ${ }^{\text {a, } d^{*}}$

${ }^{a}$ Department of Microbiology, Immunology, and Cancer Biology, ${ }^{b}$ Department of Biomedical Engineering,

${ }^{c}$ Department of Pathology, and ${ }^{d}$ Cancer Center, University of Virginia, Charlottesville, Virginia, United States of America.

*Corresponding author. Email: hz=ss@virginia.edu (Hui Zong) jks6a@virginia.edu (Jill K. Slack- Davis)

\section{Classification}

Biological Science; Genetics.

\section{Keywords}

Ovarian Cancer, Fallopian Tube, Premalignant Progression, Clonal Heterogeneity, Genetic Mosaic Mouse Model.

\section{Author Contributions}

- $\quad \mathrm{JZ}, \mathrm{JKSD}$, and $\mathrm{HZ}$ conceived the project, designed experiments.

- $\quad J Z$ performed most experiments in this manuscript.

- $\quad E C, Y J$, and BEK provided critical technical support and contributed to scientific decisions.

- $\quad$ EK performed bioinformatics analysis of mutation spectrum in human HGSOC patients.

- KAA and TJ provided the pathological assessment of the tissues.

- ACAY and KAJ performed mathematical modeling on the clonal size distribution

This PDF file includes:

Main Text

Figures 1-5 Figures S1-6 


\title{
Highlights
}

- Modeling premalignancy of fallopian tube-derived ovarian cancer with genetic mosaic.

- Dichotomous progression kinetics of initiated cells revealed by quantitative clonal analysis.

- The divergent clonal expansion is a synergic effect of cell-intrinsic property and onco-mutations.

- Highly clonogenic cells may represent fallopian tube stem/progenitor-like cells.

- Differentiation unbalance underlines early cancer progression.

\begin{abstract}
Clonal dynamics of mutant cells during early carcinogenesis result from the intersection between genetic mutations and cell-intrinsic states, and foreshadow the trajectory for further transformation. While fallopian tube (FT) Pax8+ cells are one of the origin for high-grade serous ovarian cancer (HGSOC), their clonal dynamics upon oncogenic mutations remain unknown. Here we use a mouse genetic mosaic system called MADM (Mosaic Analysis with Double Markers) that can generate sporadic mutant cells unequivocally labeled with GFP to gain access to the premalignant stages of HGSOC. The sparseness of mutant cells generated by MADM enabled us to investigate the fate of individual tumor-initiating Pax8+ cells at the clonal level. Surprisingly, we observed a dichotomous progression kinetics among mutant clones: while the vast majority stalls immediately, a small proportion expands significantly. Clonal analysis with wildtype MADM mice revealed the same dichotomy among normal Pax8+ clones, suggesting that cell-intrinsic properties determine its initial expansion capacity. Furthermore, while wildtype clones cease to expand shortly after development, expanded mutant clones manifest divergent fates: some enter quiescence, others maintained high proliferative rate and continue to progress, indicating that oncogenic mutations exaggerate clonal expansion beyond cell-intrinsic potentials. Finally, intra-clonal fate mapping showed that progressive cells have a higher propensity to maintain Pax8+ fate, implying possible prolonged stemness within progressive clones. Taken together, our studies reveal that clonal expansion during tumor initiation of HGSOC seems to be be an oncogenic mutation-induced exaggeration of a small group of Pax8+ cells with intrinsically high expansion potential, and suggest that future early detection and cancer prevention studies should focus on these rare progressing clones.
\end{abstract}




\section{Significance Statement}

Clonal dynamics of tumor-initiating cells during early carcinogenesis reflect how mutations alter cellular behaviors to establish clones susceptible for further transformation. Dissecting clonal dynamics and heterogeneity at an ostensibly normal early stage of carcinogenesis is critical for effective early detection and prevention. However, progress has been hampered by the invisibility of precancerous cells in humans and by the inability of converntional mouse models to reveal premalignant progression of cancer. Here, we developed a specialized genetic mosaic mouse model that enables tracing of the entire premalignant progression of fallopian tube-derived ovarian cancer at the clonal level. With this model, we revealed that only a small portion of fallopian tube Pax8+ cells, which likely represent the stem/progenitor-like cells in fallopian tube, can effectively initiate carcinogenesis. It would be critical to focus on these rare cells in future studies for the discovery of early detection biomarkers. Finally, our genetic mosaic mouse model can be applied in principle to many other cancer types to facilitate studies of clonal dynamics and heterogeneity during premalignant stages, shedding light on overarching principles of tumor initiation to aid cancer early detection. 


\section{Introduction}

Tumor initiates when oncogenic mutations occur in a permissive cellular context (1-3). While oncogenic mutations have been intensively studied, how mutations synergize with cell-intrinsic properties to drive tumor initiation and early progression remains elusive. Quantitative lineage tracing in mice presents a powerful tool to assess clonal dynamics of individual cells with or without mutations in their native environment, allowing one to dissect how oncomutations synergize with cell-intrinsic programs (4$6)$.

High-grade serous ovarian cancer (HGSOC) is the most prevelant and lethal (5-year survival rate, $<30 \%$ ) ovarian cancer subtype that causes about 14,000 deaths annually in the United States $(7,8)$. While malignancies affect the ovary, careful clinical studies and mouse modeling studies suggest that the fallopian tube (FT) is the origin-site for a large portion of HGSOCs (9-16). FT is a long tubal channel that connects the ovary and the uterus, a key reproductive organ where fertilization happens. FT epithelium has long been thought to consisted of ciliated cells (Acetyl-Tubulin+) that facilitate the traveling of gametes and secretory cells (Pax8+) that produce nutrition fluid (17). The secretory (Pax8+) cell has been indentified as the cancer cell-of-origin because human FT precursor lesions predominantly consist of Pax8+ cells, and moreover, introducing oncogenic mutations into FT Pax8+ cells leads to HGSOC in transgenic mice $(9,15)$. However, recent data suggest the existence of discrete populations of Pax8+ secretory cells. Daniel et al. showed that Pax8+ secretory cells present heterogeneous organoid-forming ability in vitro $(18,19)$, and Hu et al. identified multi-subsets of Pax $8+$ cells with sing-cell sequencing $(20$, 21). Whether this newly revealed diversity of Pax8+ secretory cells also present a hirarchical cellular context, and thus differential susceptibility for cancer initiation is unknown,

Here, we exploited a mouse genetic system called Mosaic Analysis with Double Markers (MADM) $(22,23)$ to trace clonal progression of individual mutated Pax8+ FT cells to compare their tumorigenic susceptibility. Meanwhile, we analyzed clonal dynamics of mutated cells and normal cells to dissect how genetic alterations synergize with intrinsic properties of certain Pax8+ cells in HGSOC initiation and early progression. Our work should provide insights for understanding HGSOC initiation in the fallopian tube and for developing effective early detection and prevention strategies. 


\section{Results}

\section{The establishment of an MADM-based mouse model to study premalignant progression of} mutated Pax8+ cells in the fallopian tube at clonal level

Mosaic Analysis with Double Markers (MADM) concomitantly generate a single GFP+ mutant cell and its RFP+ WT sibling cell from a non-labeled heterozygous mother cells through Cre/loxP-mediated inter-chromosomal mitotic recombination (Fig. 1A) $(22,23)$. The rarity of mutant cells in the tissue due to the low frequency of inter-chromosomal recombination $(0.1 \%-1 \%$ or lower) closely mimics the clonal origin of human cancer, and their immediate and unequivocal GFP-labeling enables clonal analysis right at tumor initiation $(22,24,25)$. Taking advantage of its unprecedented temporal and spatial resolution, here we establish a MADM-based mouse model for HGSOC initiation in the fallopian tube to dissect the clonal dynamics and cellular behavior of individual mutant Pax8+ cells at the pre-malignant stage.

To establish a MADM-based model for HGSOC initiation, two critical prerequisites need to be considered: 1) HGSOC-relevant tumor suppressor genes that reside on the telomeric side to the MADM cassettes; 2) a Cre transgene that is known to target the cell-of-origin for HGSOC. Based on TCGA data, we found the loss of TP53 (63\%), BRCA1/2 (78\%) and the mutations that activate the Ras-MAPK pathway (37\%, partially through loss of NF1) are among the most prevalent genetic alterations and often co-occur in human HGSOC patients (Fig. S1A) (26-30). Since TP53, BRCA1, and NF1 all reside on mouse chromosome-11 where MADM cassettes had been knocked-in (Fig. S1B) (31), we selected these mutations to drive tumor initiation. To target the cancer cell-of-origin, the FT secretory cells, between the OVGP1-CreER $(32,33)$ and the Pax8-rtTA; TetO-Cre system $(15,34,35)$, we chose the latter because CreER tends to be inefficient in catalyzing inter-chromosomal recombination in the MADM system (36). Moreover, the high level of Tamoxifen required to activate CreER for MADM labeling could confound our later data interpretation, beacause Tamoxifen is an antagonist of estrogen signaling which however is crutial for fallopian tube morphology and differentiation (37). After multi-generation breeding to incorporate all three mutant alleles and the Cre transgene into two MADM stock lines (see methods and Fig. S1C for details), we bred them together to get the MADM-mutant model to study tumor initiation and early progression (Fig. 1B). This breeding scheme also generates MADM-wildtype mice as a control (Fig. S1C). 
To assess the labeling frequency of this MADM model, we induced Cre activity in MADM-wildtype mice between postnatal day 1 to 21 (P0-21) with doxycycline (DOX) water, and found that $\sim 0.1 \%$ of $\mathrm{FT}$ epithelial cells (FTEs) were GFP+ or tdTomato+, a frequency that allows clonal tracing over time (Fig. 1C, D). Yellow cells were also found due to G2-Z segregation during mitosis or G0/G1 recombination (Fig. 1C, S2A, B). Notably, while no cells are labeled in ovary, significant number of cells in uterus were labeled by MADM, as demonstrated by previous studies (Fig. S2C) $(15,38)$. We than examined the faithfulness of our Cre system through checking the Pax8 status of labeled cells in FTs after a short-term (P19-21) Cre induction. We found nearly all MADM-labeled cells were showing Pax8+ (Fig. 1E), suggesting a high faithfulness of MADM labeling to FT Pax8+ population. To here, we have successfully established a MADM-based mouse model in which sparse, GFP-labeled mutant cells could be generated in the FT Pax8+ population to initiate HGSOC.

\section{Quantitative clonal tracing revealed divergent expansion of initiated cells}

With the sparseness and faithfulness of MADM labeling confirmed, we next evaluated clonal expansion of individual mutant cells. After inducing Cre activity between birth and weaning, we first analyzed FTs in 5-months old mice (Fig. 2A), a time when initiated cells have expanded but not yet forming histologically detectable lesions. To acquire a full view of all clones, we first cleared the FTs with the CUBIC method (39), then imaged the entire FT with light-sheet microscopy (Fig. 2B), and finally quantified the size of each clone by reconstructing image stacks. Interestingly, we found clonal sizes varied remarkably (Fig. $\mathbf{2 C}$ ): while $75 \%$ of the clones contained $<10$ cells, a small fraction of clones grew to hundreds to thousands of cells (Fig. 2D). This divergence raises the possibility that two distinct clonal expansion behaviors occurred in initiated Pax8+ cells. However, it could also merely reflect a stochastic fluctuation among equivalent clones. To tease apart these two alternatives objectively, we implemented a mathematical analysis to test whether the observed clonal size distribution follows a stochastic model of one group or includes two distinct groups. The mathematical analysis estimates the clonal size distribution from the majority clones and then asks whether the frequency of observed outlier large clones is within the prediction by the distribution. Our observed clonal sizes best fit into a negative binomial model with 50 -cells as the cutoff for outlier large clones ( $K S$ test, $p$-value $<0.1$, see methods for 
details) (40); this model predicts the observation of one clone that is larger than 50 cells. In contrast, we observed 27 clones larger than 50 cells, suggesting the existence of a significant outlier group (Fig. 2E) $\left(p<10^{-8}\right.$, Fisher Exact Test) that has higher clonal expansion potential.

To determine when these outlier clones appear, we repeated the analysis of clonal sizes with mice at one and two months of age. To our surprise, profoundly expanded clones already existed among many single-cell clones shortly after clonal induction (Fig. 2F, G). We ruled out the trivial explanation that this clonal size divergence at these young ages simply reflects their differential birth-time over the range of 21 days of DOX administration (P0-21), with data showing that prominent clonal size variance still occurred when we synchronized clonal birth with a 3-day DOX regimen (Fig. S3). We then compared the frequency of outlier large clones from 1, 2, and 5 months of age. Because the variance of clonal sizes at younger ages was smaller, a Poisson model was used to calculate the portion of outlier large clones (see methods for details). We found that the portion of outlier large clones at one month is $16.5 \pm 4.4 \%$, which increased to $28.4 \pm 1.9 \%$ at two months and held steady at $30.9 \pm 0.9 \%$ at five months (Fig. $2 \boldsymbol{H}$ ), suggesting the expansion potential of initiated Pax8+ cells was assigned at an early time point.

Collectively, these data show that initiated FT Pax8+ cells adopted distinct clonal expansion fates shortly after the oncogenic hits: with the majority immediately stalled and a small fraction profoundly expanded.

\section{The divergent expansion of initiated cells attributes to both tissue-intrinsic program and onco-} mutations

The early onset of the clonal-expansion divergence intrigues us to ask whether the initial oncogenic mutations are absolutely required for this divergence to occur. Alternatively, intrinsic heterogeneity of the clonogenic potential of Pax8+ cells could have caused this. To address this question, we induced clones in the MADM-wildtype mice, in which every clone is free of oncogenic mutations. We examined the size of these wildtype clones at 1,2, and 5 months of age and still observed evident clonal size divergence as early as one month of age (Fig. $3 A, B$ ), indicating that the clonal expansion potential of Pax8+ cells is intrinsically distinct even in the absence of mutations.

This observation further raises the question on the role of oncogenic mutations in clone expansion. To answer this question, we compared cell division in expanded wildtype clones and 
expanded mutant clones (corresponding to the outlier large clones) along a time course. With a 7-day EdU labeling right before harvest, expanded wildtype clones showed high EdU+\% at 1 month, which then dropped to very low at 2 months and 5 months. (Fig. 3C, E, F), suggesting that initial expansion capacity subsides quickly at the end of FT development (41). While the expanded mutant clones overall followed the same trend of proliferation over time, we observed two important differences: first, while proliferation decreased at 2 and 5-month, it was not as low as wildtype ones (Fig. 3E, F), suggesting that oncogenic mutations were able to sustain cell proliferation after the developmental window; second, proliferation of expanded mutant clones at 2 and 5 months ages showed a big variance, with some clones nearly as active as 1-month and others almost completed stopped (Fig. 3D, E, F, Fig. S4), suggesting that part of the expanded mutant clones were able to sustain proliferation post development. In consistent with the prolonged proliferation in expanded mutant clones, we found that, although the average size of expanded wildtype and mutant clones were similar at one-month age, the mutant clones became significantly larger at older ages (Fig. 3G), confirming a role of oncogenic mutations in prolonging proliferation in expanded mutant clones.

Taken together, we found both the the cell-intrinsically high clonogenic potential of certain Pax8+ cells and the oncogenic mutation-caused exaggeration of proliferation contribute to the formation of higly expanded mutant clones.

\section{The expanded mutant clones were spatially enriched in the distal FTs, coincide with the location of FT stem/progenitor cells.}

The high clonogenic feature showed in some of the Pax8+ cells remiscient of stem/progenitor-like cells (42). Although definitive FT stem/progenitor cell markers are lacking, several studies showed these cells are within Pax8+ population (41), and are spatially enriched in the fimbrial (distal) portion of FT (18, 19, 43, 44). To assess whether our expanded mutant clones show similar spatial preference as the FT stem/progenitor cells do, we stretched the FTs and performed whole-mount imaging to exam their location. The FT comprises three different portions: the distal portion (D) consists of the fimbria and ampulla; the middle portion $(\mathrm{M})$ consists of the isthmus; and the proximal portion $(\mathrm{P})$ consists of the uterotubal junction. Each portion can be discriminated by their distance to the ovary and their unique ductal 
morphology (Fig. 4A). Previous studies have suggested that FT stem/progenitor-like cells are eriched in the distal portion of FT Citation for the beginning.We found the expanded mutant clones almost exclusively reside in the distal FTs close to the ovary (Fig. 4B). These clones located in FT portions with "inside-out" ductal folds or with a high density of inner luminal folds, representing typical fimbria and ampulla portions, (Fig. 4C). We ruled out the possibility that such spatial preference is caused by biased generation of more mutant cells in the distal FT initially, by showing that the initial labeling frequency was similar in all segments in FTs (Fig. S5A). Notably,although the expanded mutant clones were specifically enriched in the distal FT, single-cell mutant clones still co-existed locally and accounts for the majority (Fig. 4D,E), suggesting that even within the distal FT, only a small portion of Pax8+ cells hold a high clonogenic potential.

To further investigate whether the distal enrichment of highly cologenic cells is maintained in adult FT, we induced MADM lebeling between 5-6 weeks age when the FT epithelium is considered fully developed (45), and examined one month post labeling. Consistently, we observed prominent variance of clonal size and a spatial preference of the expanded clones specifically to the distal FT (Fig. S5B). Together, these data hint that the highly expanded mutant clones may originate from the stem/progenitorlike Pax8+ subset which locates in the diatal FT.

\section{The expanded mutant clones show abnormal expansion of Pax8+ cells and fuel STICs formation.}

The epithelium of normal distal FT consists of intermixed Pax8+ cells $(30 \%)$ and ciliated cells $(70 \%)$ (Fig. S6A,B) (46). On the contrary, precursor lesions in human FT such as "secretory-celloutgrowths" (SCOUTs) and serous tubal intraepithelial carcinomas (STICs) predominantly consist of Pax8+ cells $(41,47)$. We asked whether the expanded mutant clones, as a candidate intermediate between normal and disease, could reflect this cell composition shift towards more Pax8+ cells. Indeed, we observed uninterrupted stretch of Pax8+ cells in expanded mutant clones, reminiscent of human early stage abnormality, in contrast to interspersed Pax8+ and Pax8- cells in the adjacent non-mutant regions

(Fig. 5A). As a result, the proportion of Pax8+ cells in the progressive mutant clones was significantly higher than adjacent non-mutant regions (Fig. 5B). We further hypothesized that the uninterrupted stretches of Pax8+ cells result from a biased propensity of proliferating mutant cells toward Pax8 fate. To 
test this hypothesis, we performed an EdU pulse-chase experiment to determine the fate of newly born cells within progressive clones (Fig. 5C). First, we mapped the length of EdU pulse, and found that 3-day administration led to clonal labeling of proliferating cells (Fig. S6C-G). Then we quantified clonal sizes after 4-day chase and found that, while most wildtype clones remained at 1-2 cells, many mutant clones grew much larger (Fig. S6H-J). When we examined the fate of cells in these EdU+ subclones, we found that, while $70 \%$ of cells in wildtype clones are positive for cilia, much fewer cells in mutant EdU+ subclones are ciliated (Fig.5D, E), suggesting a high propensity of mutant cells to adopt a Pax8+ cell fate.

We then asked whether the long-termly progressive mutant clones are capable to form FT lesions, for example STICs. We examined a total of 83 mice across an age range with H\&E stained FT sections, and identified 6 GFP+ STICs among 40 mice aged 8-14 months (Fig. 5F,G), suggesting that the expanded mutant clones are indeed capable for further progression to STICs. The rareness of STICs was expected in the MADM model, because 1)different from conditional knockout models, MADM only generates very few mutant cells initially; 2)among the initial mutant cells, only a small portion are capable for expansion (Fig. 2D); 3)among the initially expanded clones, less than half of them maintain long-term proliferation (Fig. 3E, F); 4)we suspect that not every proliferative clone can eventually progress to STICs. While desirable, we could not wait longer to assess whether ovarian tumor could form due to the formation of invasive uterine tumor at around 1 year of age, as uterine epithelium initially were also hitted by oncogenic mutations (Fig. S2C). In summary, the expanded mutant clones are capable for further progression and recapitulate early abnormalities observed in human.

\section{Discussion}

In this study, we utilized a mouse genetic mosaic system to study clonal dynamics during tumor initiation and premalignant progression of HGSOC. Taking advantage of the clonal resolution afforded by the model, we traced progression of individual initiated cells and found that they immediately adopt dichotomous fates right after the acquisition of oncogenic mutations: while most clones stall, a small number of them expand extensively, likely due to the distinction of cell-intrinsic properties among Pax8+ cells. Furthermore, while wildtype clones cease to expand shortly after development, expanded mutant clones had divergent fates: while some enter quiescence, others maintained high proliferative rate and 
continue to progress, indicating that oncogenic mutations exaggerate clonal expansion beyond cellintrinsic potentials. Finally, intra-clonal fate mapping showed that progressive cells have a higher propensity to maintain Pax8+ fate, implying possible prolonged stemness within progressive clones. Taken together, our studies reveal that clonal expansion during tumor initiation of HGSOC seems to be an oncogenic mutation-induced exaggeration of a small group of cells with intrinsic expansion potential.

\section{MADM as a tool to reveal clonal dynamics during tumor initiation and early progression}

Although understanding clonal dynamics of mutated cells at the initiation and premalignant stages of HGSOC is integral for developing effective early detection and prevention strategies, it remained elusive due to the lack of patient samples from this early stages (i.e., prior to the development of pathologically recognizable lesions) and a shortage of mouse models that recapitulate premalignant progression. The MADM-based cancer mouse model we developed here generates rare, GFP-labeled mutant cells for cancer initiation, which not only provides access to the premalignant stages of cancer, but also allows us to study progression of individual initiated cells at the clonal level (24). This approach to model cancer initiation and premalignancy, in principle, can be applied to any other cancer type and should present a powerful tool to dissect dynamics and heterogeneity of cancer initiating cells during early carcinogenesis at with single-cell and clonal resolution. The analytic power of the MADM-based model is further enhanced by an internal control, i.e., RFP-labeled wildtype sibling clones, and by MADM-wildtype control mice, which are critically useful for dissecting the role of onco-mutations versus cell inherent lineage programs in cancer initiation (48). When combined with EdU pulse-chase assays, one could further trace the behavior of actively proliferating cells within progressing mutant clones, providing a realtime assessment of the distribution pattern and cellular fate of cells that fuels clone growth and progression (6).

\section{The intersection between cell intrinsic properties and oncogenic mutations underpins the expansion potential of initiated cells.}

Both the genetic lesions and the inherent lineage program of the cell-of-origin play major roles in determining clonal dynamics at the malignant stage $(1,49,50)$. Our study of tumor initiating Pax $8+$ cells 
at a premalignant stage seems to point to the intrinsic cellular properties as the determinant of their dichotomous expansion fate. Mutations played a role in exaggerating the expansion and enabling longterm persistence of the cells predesignated with high expansion potential; however, they did not determine the initial expansion fate. The divergent clonal expansion of mutant Pax8+ cells we observed was initially hypothesized to be caused by differentially acquired extra mutations. However, we later found wildtype clones, which are unlikely to acquire extra mutations, also showed divergent clonal expansion, suggesting that mutations are not the cause. Instead, the inherent program of fallopian tube, likely the intrinsic cellular heterogeneity of Pax8+ cells led to the divergent clonal expansion. Indeed, recent single cell sequencing data of fallopian tube epithelia revealed multiple subsets of Pax $8+$ cells. An early secretory subset characterized by increased expression of stem/progenitor-related regulons (MYC and NR2F2) was identified (21), which may correspond to the highly progressive Pax8+ cells we found. Other studies demonstrated heterogeneous organoid-forming ability of Pax8+ cells under the same condition, with those highly potent cells specifically enriched in the distal portion of fallopian tube $(18,43)$, which is consistent with the location of our highly expanded mutant clones. Together, we argued that the intrinsic cellular heterogeneity of Pax8+ cells most likely underpins the divergent clonal expansion of initiated cells.

The distal FT preferences of the highly clonogenic Pax8+ cells we described here and the enrichedment of organoid-forming cells in this region reported, coincide with the hotspot of human FT precursor lesions found in clinical studies $(14,51,52)$. This distal FT preference of precursor lesions in human is thought to be due to their proximity to the ovary and therefore repeated exposure to ovulation released inflammatory cytokines, which lead to DNA damage (53-56). However, we observed the distal FT preferences of the highly expanded mutant clones as early as one month of age, a time when the mouse has experienced limited to no ovulation $(57,58)$. Moreover, the distal FT preferences of the organoid-forming cells was found with an in vitro system devoid of ovulation effects. These data suggest an alternative but not mutually exclusive hypothesis to explain the distal FT preference of precursor lesions: a Pax8+ subset predesignated with high malignant susceptibility are inherently enriched in the distal end. 


\section{Implications of premalignant heterogeneity for cancer early-detection and prevention studies}

Although the exact differences between the highly progressive and stalled Pax8+ cells need to be further explored, the clonal dynamics revealed by our study carries important implications for early detection and prevention studies of HGSOC. There seems to be a process of gradual attrition among mutant Pax8+ cells: initially, vast majority of mutant cells stall while only a small portion expand; then only part of those initially expanded mutant cells could sustain their progressiveness over time; moreover, within the rare, constantly progressing mutant clones, only a small portion of the cells maintain proliferation. To identify precise early detection markers, we need to focus on the signatures from the rare proliferating cells within those rare, constantly progressing mutant clones. General profiling of all mutant cells would bring in tremendous noise from stalled mutant cells and from mutant clones that initially expanded but lose progression later. Markers identified from overly generalized profiling likely would lead to over-diagnosis. For the same reason, efficacy- measurement of cancer prevention studies should also focus on these progressing cells rather than the bulk mutant clones that are already at their dead end.

In summary, our study demonstrated the usage of a mouse genetic mosaic system to dissect clonal dynamics of individual initiated mutant cells at an unprecedentedly early stage with single-cell resolution. Utilizing this model, we characterized the cellular and spatial heterogeneity of cancer-initiating cells for FT-derived ovarian cancer, which should pave the way for discovery of more precise early detection markers and preventive therapeutics. 


\section{Materials and Methods}

\section{Animals}

All animal procedures are approved by the Institutional Animal Care and Use Committee (IACUC) at the University of Virginia in accordance with national guidelines to ensure the humanity of all animal experiments. The following mouse lines were crossed to establish the MADM-mutant and MADM-wildtype mice: TG11ML (stock NO. 022976 JAX), GT11ML (stock NO. 022977 JAX), BRCA1 $1^{\text {flox }}$ (strain NO. 01XC9 $\mathrm{NCl}$ ), NF1 $1^{\text {flox }}$ (strain no. 01XM4; NCI), TP53 ${ }^{K O}$ (stock no. 002101; JAX), PAX8-rtTA (stock NO. 007176 JAX)(34), TetO-Cre (stock NO. 006234 JAX)(35).

\section{In vivo drug delivery}

Doxycycline was administered through the drinking water $(2 \mathrm{mg} / \mathrm{ml})$ of mom and transmitted to MADM pups through milk (P0-P21) to induce Cre recombinase. 5-ethynyl-2'-deoxyuridine (EdU) (Invitrogen, Cat\# A10044) was administered through drinking water $(0.5 \mathrm{mg} / \mathrm{ml})$. EdU staining was performed following standard procedures (59).

\section{Immunostaining}

Reproductive tracts of MADM mice were harvested then fixed overnight with cold $4 \%$ paraformaldehyde (PFA) at $4^{\circ} \mathrm{C}$. Tissues were then washed with PBS to remove recessive PFA, soaked with $30 \%$ sucrose, embedded in optimal cutting temperature $(\mathrm{OCT})$, and stored at $-80^{\circ} \mathrm{C}$. The tissues were sectioned at $18 \mu \mathrm{m}$ thickness. For staining, slides were incubated in Permeabilization/blocking buffer $(0.3 \%$ Triton-X 100 in PBS [PBT] plus 5\% normal donkey serum) for 20 min then primary antibodies (Pax8,1:50,10336-1AP, Proteintech; AcTUB,1:500, T7451, Sigma) diluted in Permeabilization/block buffer were added and incubated at $4^{\circ} \mathrm{C}$ overnight. Secondary antibody incubation was performed for 1 hour at RT in PBT. To stain nuclei, slides were incubated in DAPI solution $(1 \mathrm{ug} / \mathrm{mL}$ in PBT) for $5 \mathrm{~min}$ before being mounted with $70 \%$ glycerol and covered. Fluorescent images were acquired on Zeiss LSM 700/710 confocal microscope. Images were processed with Zen and Fiji.

\section{Tissue clearing with CUBIC method and 3D imaging}

The PFA fixed ovary and fallopian tube were cleared for large-scale 3D imaging with the standard CUBIC method (39). Tissues were first immersed in $50 \%$ reagent- 1 and shaken at $110 \mathrm{rpm} 37^{\circ} \mathrm{C}$ for $6 \mathrm{~h}$ and then transferred to $100 \%$ reagent-1 with DAPI $(1 \mathrm{ug} / \mathrm{ml})$ to shake for $2-3$ days until becoming transparent. After 
reagent-1, tissues were washed three times with PBS, 30 mins each time with shaking to remove the reagent-1. Tissues were immersed in $50 \%$ reagent- 2 for $6 \mathrm{~h}$ with shaking at $37^{\circ} \mathrm{C}$; thereafter, buffer was exchanged to $100 \%$ reagent- 2 to shake for $24 \mathrm{~h}$.

The Zeiss Z.1 light-sheet microscopy system was used for acquiring images. Tissues were embedded in $2 \%(w / w)$ agarose (LE quick dissolve agarose, GeneMate E-3119-500) gel; the agarose was dissolved in a modified reagent-2 $\left(10 \%\right.$ urea, $50 \%$ sucrose, $30 \% \mathrm{H}_{2} \mathrm{O}$, hot stir at $80^{\circ} \mathrm{C}$ until fully dissolved, then add $10 \%$ Tri-ethanolamine). The warm agarose gel solution together with tissue was aspirated into a $1 \mathrm{ml}$ syringe with the neck cut off, and then the syringe was placed on ice for a quick solidification. The syringe was placed on the holder of the light-sheet microscope, and the tissue was pushed out for imaging.

\section{Counting clone size}

Whole FT were cleared and imaged at 5 um intervals with light-sheet microscopy at single-cell resolution through the entire tissue. Three-dimensional reconstruction of clones was performed; labeled cells that were found at the exact location through multiple image stacks were counted as one clone. The size of clones was measured manually by counting the DAPI-stained nuclei.

\section{Mathematical detection of outlier clones.}

The clonal size distribution along the fallopian tube was evaluated in terms of cell division events. With $\mathrm{n}$ as the number of cells per clone, there will be $\mathrm{n}-1$ cell divisions. For clonal size distribution at five months, we fitted with a negative binomial distribution, which captures the behavior of the overdispersed data observed in our mice models, by allowing the mean and variance to be different. Clonal size distribution at 1 and 2 months, which showed a smaller range, were fitted with a Poisson distribution. To estimate the statistical significance of our fitting, we generated null distributions for the Kolmogorov-Smirnov (KS) statistic between an ideal probability mass function and a random negative binomial/Poisson distribution over 1.000 iterations. The parameters used for generating the null distributions were obtained by merging all datasets and taking a maximum of 44/8-9-10, which showed significance in our first general screening. Then, the empirical nominal p-value of the observed KS for the merged data was calculated relative to the null distribution until a cutoff with significance was observed. Our final cutoff was set as the maximum number of divisions for which the probability of observing our KS was $p>=0.1$. The validity of this cutoff was confirmed by evaluating the individual datasets over the null distributions. Then, the maximum 
expected clonal sizes $(\mathrm{n})$ in the fallopian tube were calculated, and any clone with a size larger than $\mathrm{n}$ was taken as the outlier. Subsequently, we computed the expected clone sizes for all the models, and we tested their significance against the observed values in our datasets using Fisher exact/Chi-square tests.

Finally, we defined over-proliferative clones (Expected - observed outliers) for the size distributions observed under the Poisson model and tested their significance between 1, 2, and 5 months by an Anova with Tukey's Honest Significant Difference test.

\section{Acknowledgments}

We thank Dr. Zhe Li, Xiaoyu Zhao and Bing Xu for providing critical feedback on the manuscript and Joceylyn Ray for the artwork of the fallopian tube cartoon. We also thank Dr. Stacey Criswell at the Advanced Microscopy Facility, Dr. Pat Pramoonjago at the Biorepository and Tissue Research Facility, Sheri Vanhoose at the Research Histology Core, and Shelly Verling at the vivarium for their assistance on the project. These core facilities are supported by UVA Cancer Center grant \#P30CA044579. We are grateful to Dr. Ammasi Periasamy and Dr. Ruofan Cao at the Keck Center for the usage of the Lightsheet Z.1 microscopy system. This work was partly supported by the Department of Defense Ovarian Cancer Research Program \#W81XWH-17-1-0174 (J.K.S-D. \& H.Z.), the Rivkin Center (H. Z.), the UVA Cancer Center Seed Grant (J.K.S-D. \& H.Z.), and the UVA Cancer Center Training Grant (J.Z.). 


\section{References}

1. Visvader JE (2011) Cells of origin in cancer. Nature 469(7330):314-322.

2. Barker N, et al. (2009) Crypt stem cells as the cells-of-origin of intestinal cancer. Nature 457(7229):608-611.

3. Chen W, et al. (2008) Malignant transformation initiated by Mll-AF9: gene dosage and critical target cells. Cancer Cell 13(5):432-440.

4. Alcolea MP, et al. (2014) Differentiation imbalance in single oesophageal progenitor cells causes clonal immortalization and field change. Nat Cell Biol 16(6):615-622.

5. Jin S (2019) Bipotent stem cells support the cyclical regeneration of endometrial epithelium of the murine uterus. PNAS 116(14):6848-6857.

6. Driessens G, Beck B, Caauwe A, Simons BD, \& Blanpain C (2012) Defining the mode of tumour growth by clonal analysis. Nature 488(7412):527-530.

7. Siegel RL, Miller KD, \& Jemal A (2020) Cancer statistics, 2020. CA Cancer J Clin 70(1):7-30.

8. Vaughan $S$, et al. (2011) Rethinking ovarian cancer: recommendations for improving outcomes. Nat Rev Cancer 11(10):719-725.

9. Karnezis AN, Cho KR, Gilks CB, Pearce CL, \& Huntsman DG (2017) The disparate origins of ovarian cancers: pathogenesis and prevention strategies. Nat Rev Cancer 17(1):65-74.

10. Keren Levanon CC, Ronny Drapkin (2008) New insights into the pathogenesis of serous ovarian cancer and its clinical impact. J Clin Oncol 26(32):5284-5293.

11. Fabiola Medeiros MGM, Yonghee Lee, Julia A Elvin, Michael J Callahan, Colleen Feltmate, Judy E Garber, Daniel W Cramer, Christopher P Crum (2006) The tubal fimbria is a preferred site for early adenocarcinoma in women with familial ovarian cancer syndrome。. The American journal of surgical pathology 30(2):230-236.

12. Press JZ, et al. (2008) Ovarian carcinomas with genetic and epigenetic BRCA1 loss have distinct molecular abnormalities. BMC Cancer 8:17.

13. Barakat RR FM, Saigo PE, Robson ME, Offit K, Boyd J. (2000) Absence of premalignant histologic, molecular, or cell biologic alterations in prophylactic oophorectomy specimens from BRCA1 heterozygotes. Cancer 89(2):383-390.

14. Lee $\mathrm{Y}$, et al. (2007) A candidate precursor to serous carcinoma that originates in the distal fallopian tube. J Pathol 211(1):26-35.

15. Perets R, et al. (2013) Transformation of the fallopian tube secretory epithelium leads to high-grade serous ovarian cancer in Brca;Tp53;Pten models. Cancer Cell 24(6):751-765.

16. Labidi-Galy SI, et al. (2017) High grade serous ovarian carcinomas originate in the fallopian tube. Nat Commun 8(1):1093.

17. Stewart CA \& Behringer RR (2012) Mouse Oviduct Development. Mouse Development: From Oocyte to Stem Cells, ed Kubiak JZ (Springer Berlin Heidelberg, Berlin, Heidelberg), pp 247-262.

18. Daniel Y. Paik DMJ, Amanda M. Schafenacker, Victor S. Velasco, May S. Shung, Donghui Cheng, Jiaoti Huang, Owen N. Witte, and Sanaz Memarzadeh. (2012) Stem-like epithelial cells are concentrated in the distal end of the fallopian tube: a site for injury and serous cancer initiation. Stem Cells 30(11):2487-2497.

19. Rose IM, et al. (2020) WNT and inflammatory signaling distinguish human Fallopian tube epithelial cell populations. Sci Rep 10(1):9837. 
20. Zhiyuan Hu MA, Abdulkhaliq Alsaadi,Nina Wietek,Matteo Morotti, Tingyan Shi, Zhe Zhong, Laura Santana Gonzalez, Salma El-Sahhar, Mohammad Karami,NejadRanjbar, Garry Mallett, Yun Feng, Kenta Masuda,Yiyan Zheng, Kay Chong, Stephen Damato, Sunanda Dhar, Leticia Campo,Riccardo Garruto Campanile,Hooman Soleymani majd,Vikram Rai,David Maldonado-Perez, Stephanie Jones, Vincenzo Cerundolo, Tatjana Sauka-Spengler, Christopher Yau, and Ahmed Ashour Ahmed. (2020) The Repertoire of Serous Ovarian Cancer Non- genetic Heterogeneity Revealed by Single-Cell Sequencing of Normal Fallopian Tube Epithelial Cells. Cancer Cell (37):226-242.

21. Dinh $\mathrm{HQ}$, et al. (2021) Single-cell transcriptomics identifies gene expression networks driving differentiation and tumorigenesis in the human fallopian tube. Cell Rep 35(2):108978.

22. Zong H, Espinosa JS, Su HH, Muzumdar MD, \& Luo L (2005) Mosaic analysis with double markers in mice. Cell 121(3):479-492.

23. Mandar Deepak Muzumdar 1 LL, Hui Zong (2007) Modeling sporadic loss of heterozygosity in mice by using mosaic analysis with double markers (MADM). Proc Nati Acad Sci USA 104(11):4495-4500.

24. Liu C, et al. (2011) Mosaic analysis with double markers reveals tumor cell of origin in glioma. Cell 146(2):209-221.

25. Hippenmeyer S (2013) Dissection of gene function at clonal level using mosaic analysis with double markers. Frontiers in Biology 8(6):557-568.

26. Walsh T CS, Lee MK, Pennil CC, Nord AS, Thornton AM, Roeb W, Agnew KJ, Stray SM, Wickramanayake A, Norquist B, Pennington KP, Garcia RL, King MC, Swisher EM. (2011) Mutations in 12 genes for inherited ovarian, fallopian tube, and peritoneal carcinoma identified by massively parallel sequencing. Proc Nati Acad Sci USA 108(44):1803218037.

27. Anonymous (2011) Integrated genomic analyses of ovarian carcinoma. Nature 474(7353):609-615.

28. Ahmed AA, et al. (2010) Driver mutations in TP53 are ubiquitous in high grade serous carcinoma of the ovary. J Pathol 221(1):49-56.

29. Patch AM, et al. (2015) Whole-genome characterization of chemoresistant ovarian cancer. Nature (1476-4687 (Electronic)).

30. Angela Toss CT, Elisabetta Razzaboni,Giannina Contu,Giovanni Grandi,Angelo Cagnacci,Russell J. Schilder, and Laura Cortesi. (2015) Hereditary Ovarian Cancer: Not Only BRCA 1 and 2 Genes. Biomed Res Int (341723).

31. Tasic B, et al. (2012) Extensions of MADM (mosaic analysis with double markers) in mice. PLoS One 7(3):e33332.

32. Sherman-Baust CA, et al. (2014) A genetically engineered ovarian cancer mouse model based on fallopian tube transformation mimics human high-grade serous carcinoma development. The Journal of Pathology 233(3):228-237.

33. Wu R, et al. (2016) Impact of oviductal versus ovarian epithelial cell of origin on ovarian endometrioid carcinoma phenotype in the mouse. J Pathol 240(3):341-351.

34. Traykova-Brauch $M$, et al. (2008) An efficient and versatile system for acute and chronic modulation of renal tubular function in transgenic mice. Nat Med 14(9):979-984. 
35. Anne-Karina T Perl SEW, Andras Nagy, Corrinne G Lobe, Jeffrey A Whitsett (2002) Early restriction of peripheral and proximal cell lineages during formation of the lung. PNAS 99(16):10482-10487.

36. Gao P, et al. (2014) Deterministic progenitor behavior and unitary production of neurons in the neocortex. Cell 159(4):775-788.

37. A Okada YO, SL Brody, H Watanabe, A Krust, P Chambon, and T Iguchi. (2004) Role of foxj1 and estrogen receptor alpha in ciliated epithelial cell differentiation of the neonatal oviduct. Journal of Molecular Endocrinology 32(3):615-625.

38. Bowen NJ, et al. (2007) Emerging roles for PAX8 in ovarian cancer and endosalpingeal development. Gynecol Oncol 104(2):331-337.

39. Susaki EA, et al. (2015) Advanced CUBIC protocols for whole-brain and whole-body clearing and imaging. Nat Protoc 10(11):1709-1727.

40. Fisher CIBaRA (June 1953) Fitting the Negative Binomial Distribution to Biological Data. Biometric 9(2):176-200.

41. Ghosh A, Syed SM, \& Tanwar PS (2017) In vivo genetic cell lineage tracing reveals that oviductal secretory cells self-renew and give rise to ciliated cells. Development 144(17):3031-3041.

42. Elaine Fuchs JAS (2000) Stem Cells. Cell 100(1):143-155.

43. Xie Y, Park ES, Xiang D, \& Li Z (2018) Long-term organoid culture reveals enrichment of organoid-forming epithelial cells in the fimbrial portion of mouse fallopian tube. Stem Cell Res 32:51-60.

44. Wang Y, et al. (2012) Identification of quiescent, stem-like cells in the distal female reproductive tract. PLoS One 7(7):e40691.

45. Yamanouchi H, Umezu T, \& Tomooka Y (2010) Reconstruction of oviduct and demonstration of epithelial fate determination in mice. Biol Reprod 82(3):528-533.

46. Harwalkar K, et al. (2021) Anatomical and cellular heterogeneity in the mouse oviductits potential roles in reproduction and preimplantation developmentdagger. Biol Reprod 104(6):1249-1261.

47. Chen EY, et al. (2010) Secretory cell outgrowth, PAX2 and serous carcinogenesis in the Fallopian tube. J Pathol 222(1):110-116.

48. Phillippe P. Gonzalez JK, Rui Pedro Galvao,Nichola Cruickshanks, Roger Abounader,Hui Zong. (2018) p53 and NF 1 loss plays distinct but complementary roles in glioma initiation and progression. Glia 66(5):999-1015.

49. Greaves M \& Maley CC (2012) Clonal evolution in cancer. Nature 481(7381):306-313.

50. Lauren M.F. Merlo JWP, Brian J. Reid \& Carlo C. Maley (2006) Cancer as an evolutionary and ecological process. Nature Reviews Cancer 6:924-935.

51. Fabiola Medeiros 1 MGM, Yonghee Lee, Julia A Elvin, Michael J Callahan, Colleen Feltmate, Judy E Garber, Daniel W Cramer, Christopher P Crum (2006) The tubal fimbria is a preferred site for early adenocarcinoma in women with familial ovarian cancer syndrome. The American journal of surgical pathology 20(2):230-236.

52. Mingels MJ, et al. (2012) Tubal epithelial lesions in salpingo-oophorectomy specimens of BRCA-mutation carriers and controls. Gynecol Oncol 127(1):88-93. 
53. Che-Fang Hsu P-CC, Vaishnavi Seenan, Dah-Ching Ding and Tang-Yuan Chu. (2021) Ovulatory Follicular Fluid Facilitates the Full Transformation Process for the Development of High-Grade Serous Carcinoma. Cancers 13(3):468.

54. Murdoch WJ (2008) Ovulatory factor in ovarian carcinogenesis. Adv Exp Med Biol. 622:119-128.

55. Huang HS, et al. (2015) Mutagenic, surviving and tumorigenic effects of follicular fluid in the context of p53 loss: initiation of fimbria carcinogenesis. Carcinogenesis 36(11):14191428.

56. King SM, et al. (2011) The impact of ovulation on fallopian tube epithelial cells: evaluating three hypotheses connecting ovulation and serous ovarian cancer. Endocr Relat Cancer 18(5):627-642.

57. TimothyJ. Safranski WRL, Duane H. Keisler. (1993) Correlations among Three Measures of Puberty in Mice and Relationships with Estradiol Concentration and Ovulation. Biology of Reproduction 48(3):669-673.

58. Gaytan F, et al. (2017) Development and validation of a method for precise dating of female puberty in laboratory rodents: The puberty ovarian maturation score (Pub-Score). Sci Rep 7:46381.

59. Adrian (2008) A chemical method for fast and sensitive detection of DNA synthesis in vivo. PNAS 105(7):2415-2420. 


\section{Figure 1}

A

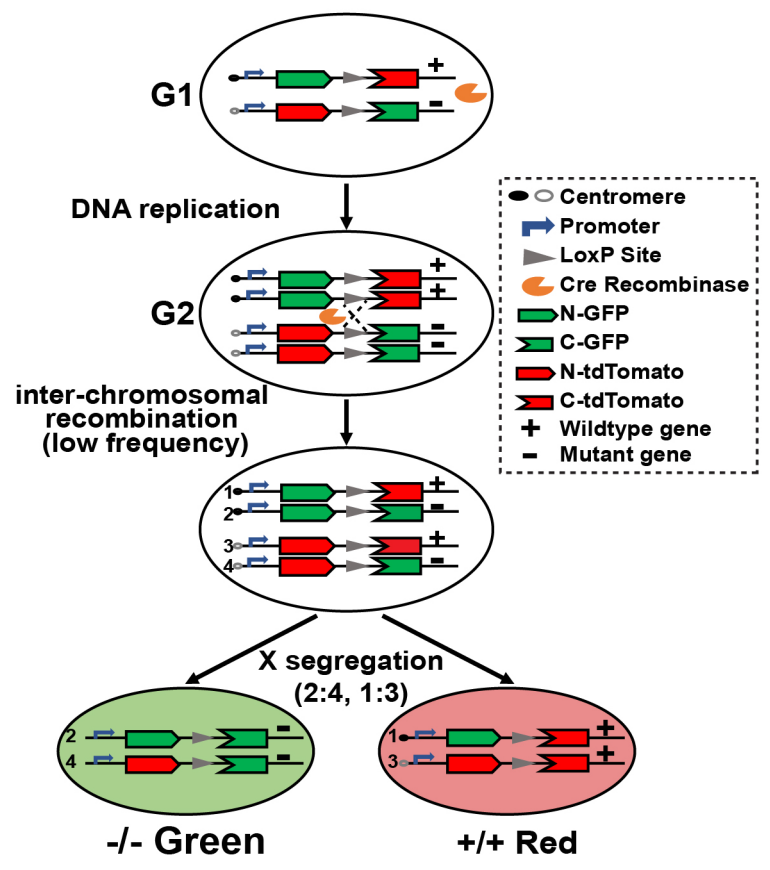

C
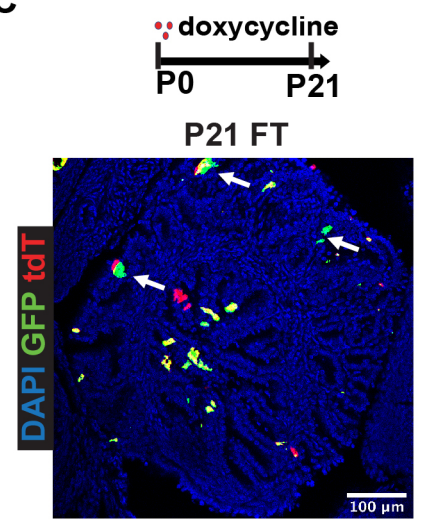

D

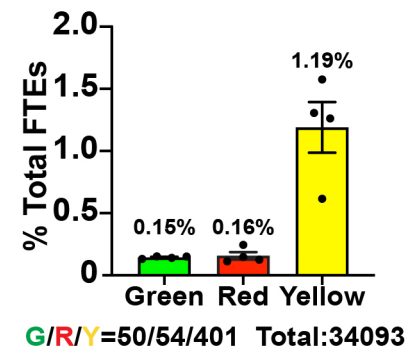

B

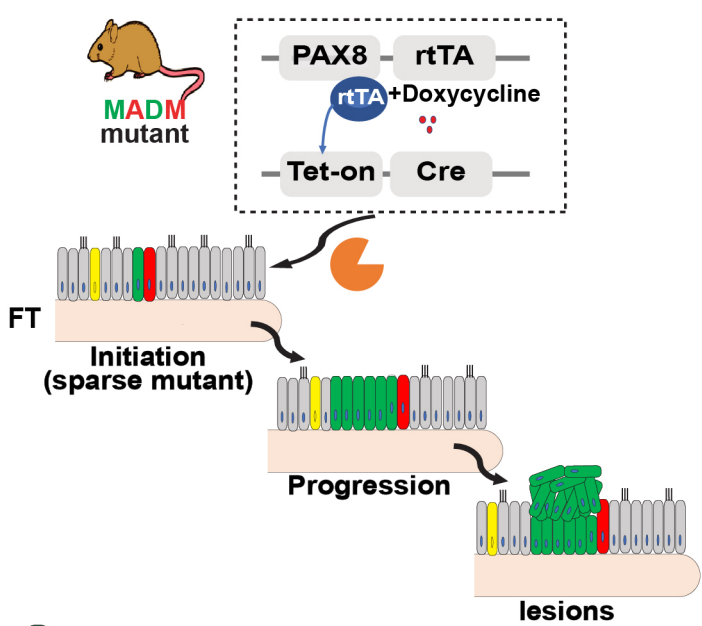

Mutant: TP53,BRCA1,NF1-KO

Wildtype .0. Heterozygous

E
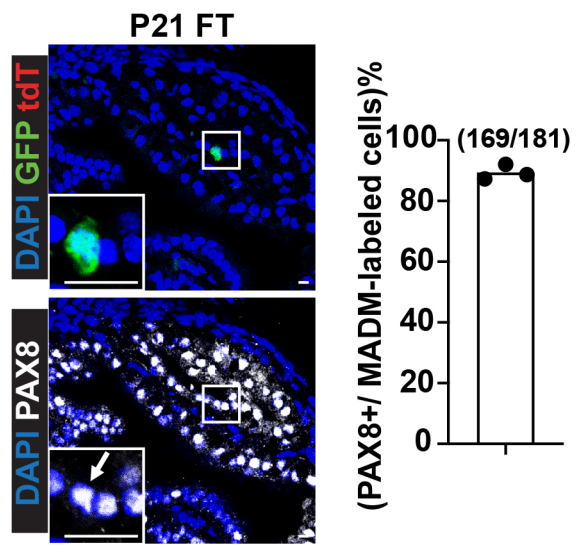
Figure 1. Establishment of an MADM-based mouse model for clonal tracing of initiated FT cells.

(A) MADM mechanism: MADM contains a pair of knock-in cassettes of chimeric GFP and tdTomato coding sequences, separated by a loxP-containing intron. Cremediated inter-chromosomal recombination in mitotic cells followed by $X$ segregation generates singly colored cells with altered genotype. GFP expressing (Green) cells are homozygous mutant, tdTomato expressing (Red) cells are wildtype.

(B) The scheme of MADM-based modeling of HGSOC initiation in the FT.

(C) MADM labeled cells in FT after inducing Cre between post-natal day 0 and day 21 (P0-21).

(D) Green, red and yellow cell portion among all FT epithelial cells $( \pm \mathrm{SEM}, n=3)$.

(E) Left panel: MADM-labeled cells were stained positive for Pax8. Right panel: quantification of Pax8+\% among all MADM-labeled cells. $(n=3)$. Scale bar= $=50 \mu \mathrm{m}$. 


\section{Figure 2}

A

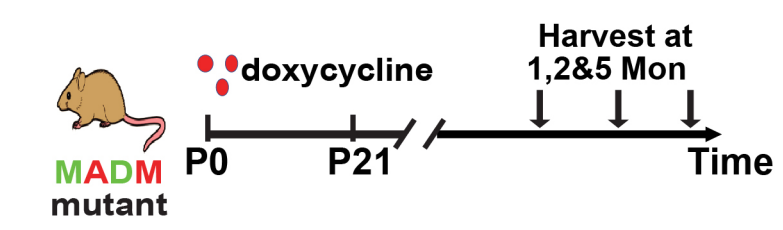

B

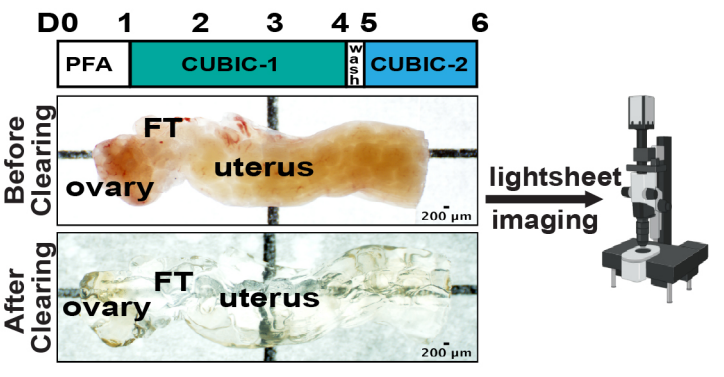

E

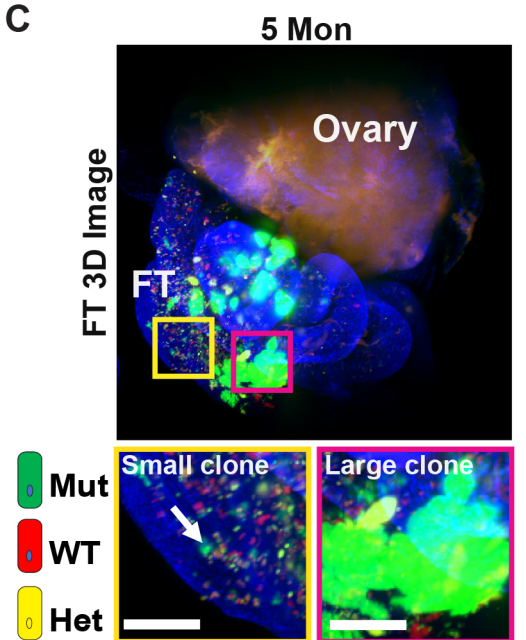

F

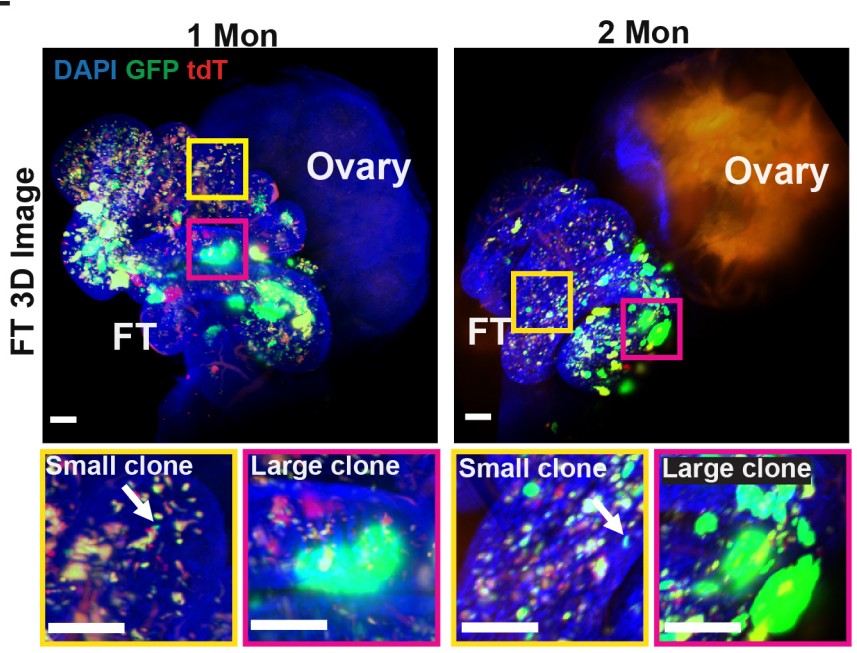

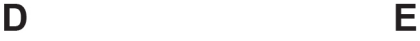
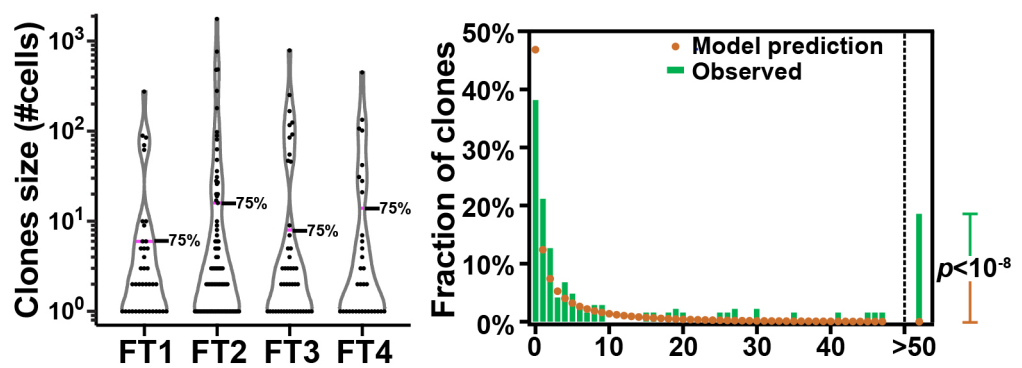

G

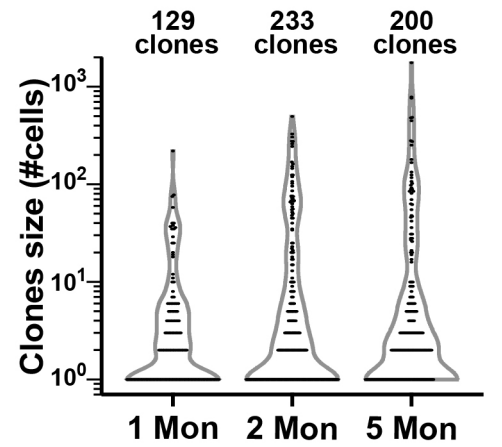

H

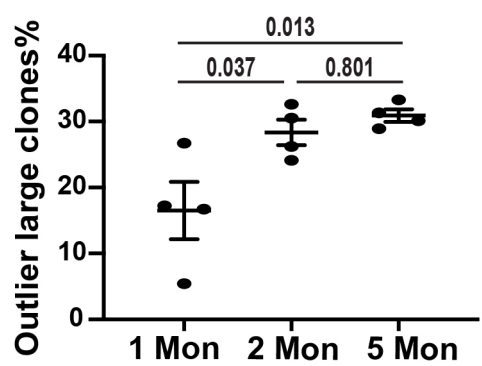


Figure 2: Quantitative clonal tracing revealed divergent expansion of initiated cells.

(A) The scheme of DOX treatment and FT harvesting for measuring mutant clone size

(B) The pipeline for high-resolution whole-FT imaging: fixed reproductive tracts were cleared though the CUBIC method, then 3D imaged with light-sheet microscopy.

(C) Representative 3D light-sheet images of cleared whole FT from 5 months old MADM-mutant mice $(n=4)$. The yellow box shows non-expanded clones, the magenta box shows expanded clones. Scale bar=50 $\mu \mathrm{m}$.

(D) Violin plot of clonal size distribution of mutant clones in four independent FTs from mice at 5 months age.

(E) Fit of the observed clonal size distribution of mutant clones at 5 months to a negative binomial model. Bars show observed data, points show model prediction. Number of clones $>50$ cells observed is significantly higher than prediction. Fisher Exact Test, $p<10^{-8}$.

(F) Representative 3D light-sheet images of cleared whole FT from 1 and 2 months old MADM-mutant mice $(n=4)$. The yellow box shows non-expanded clones, the magenta box shows expanded clones. Scale bar=50 $\mu \mathrm{m}$.

(G) Violin plot of clonal size distribution of mutant clones at 1 and 2 months. Data pooled from 4 mice.

(H) The portion of profoundly-expanded outlier clones detected at 1,2,5 months. $( \pm$ SEM, $n=4$, Anova test with Tukey HSD) 


\section{Figure 3}

A

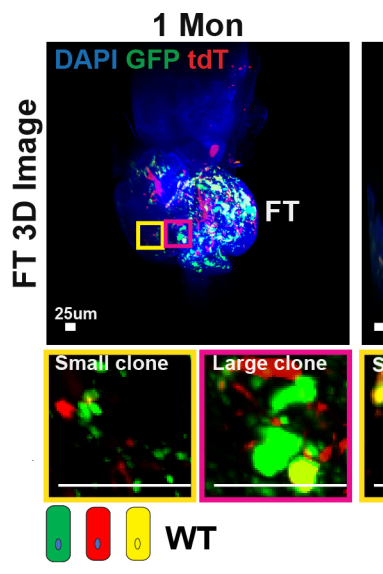

C

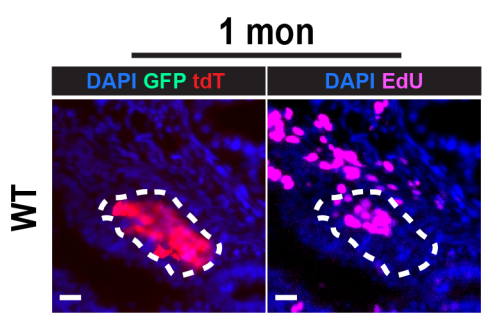

D
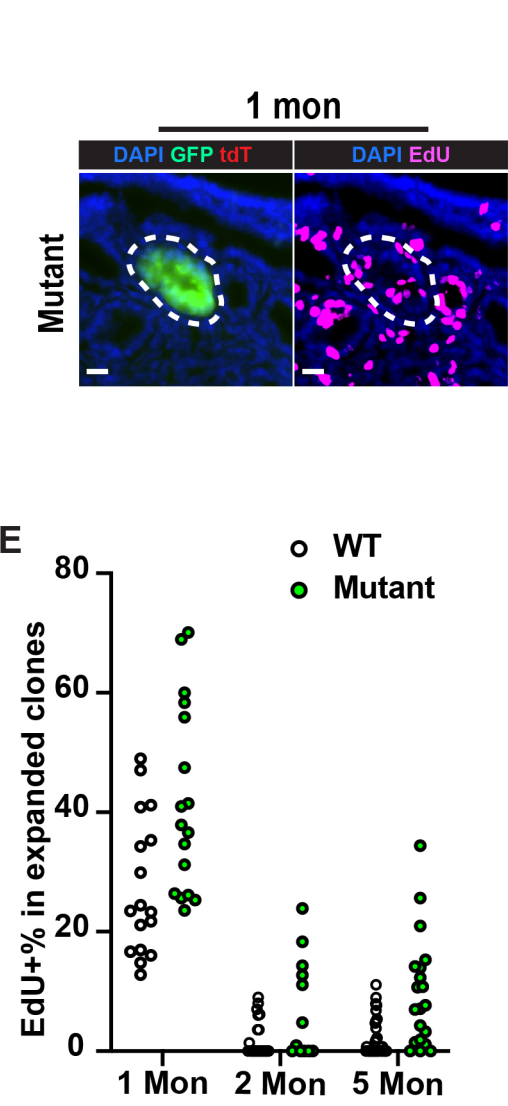

2 Mon
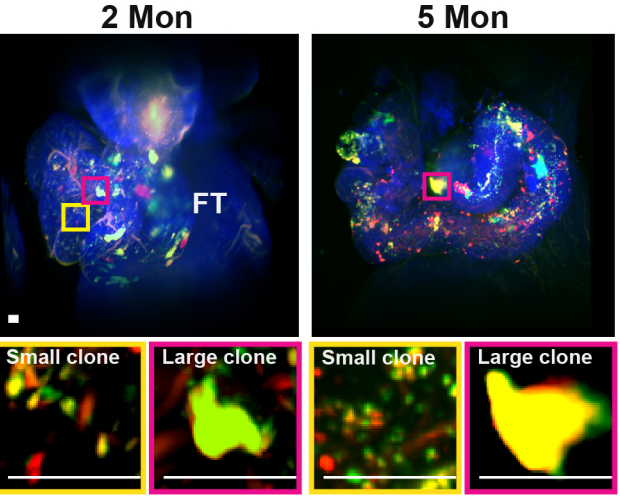

2 mon

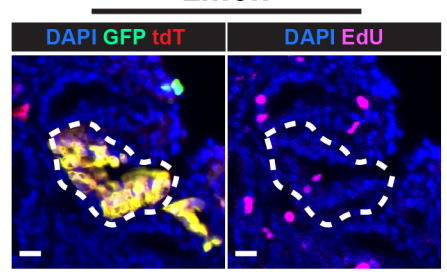

2 mon

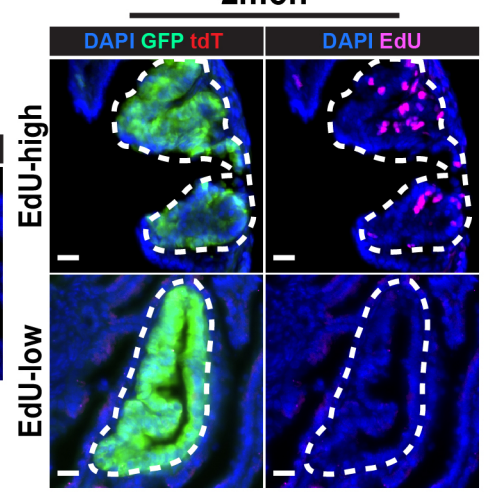

F

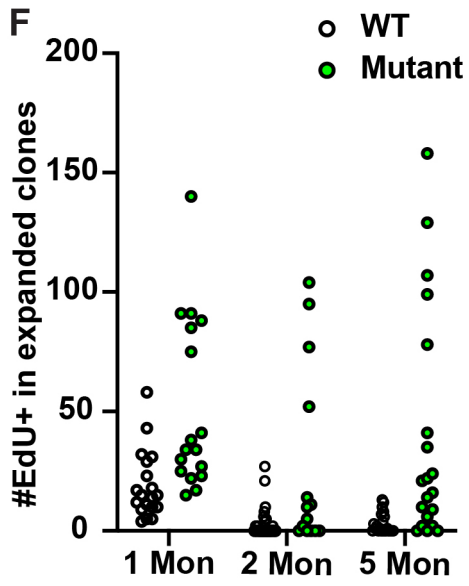

B
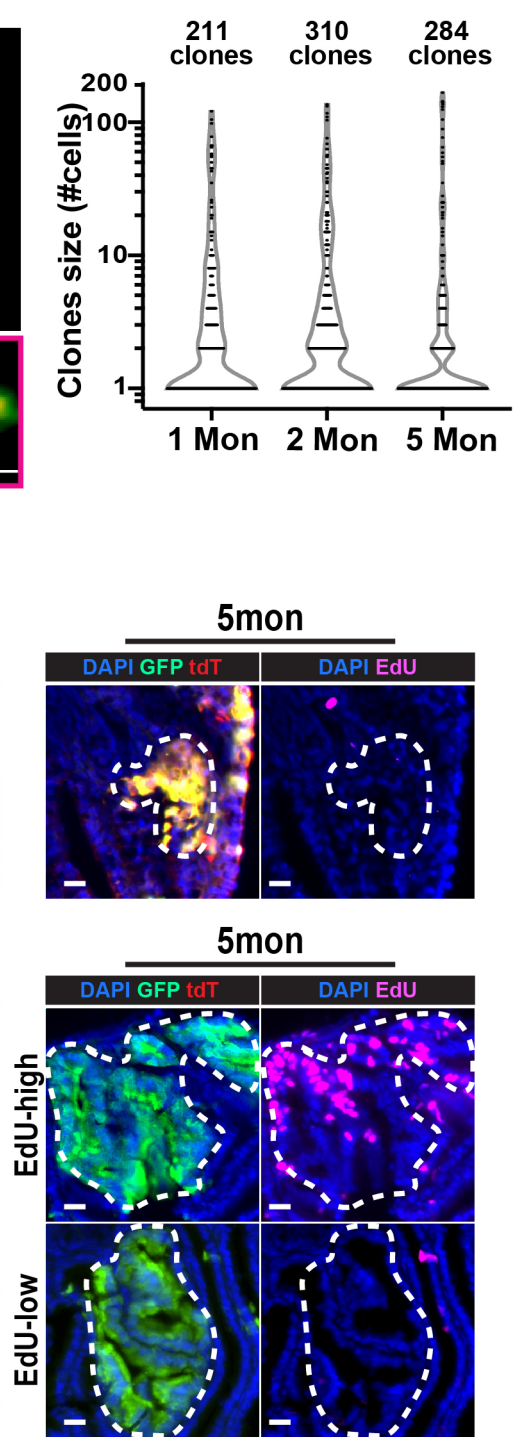

G

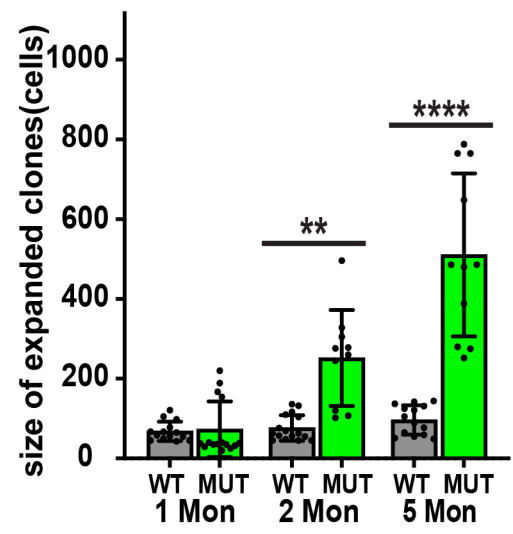


Figure 3: The divergent expansion of initiated cells attributes to both tissueintrinsic program and onco-mutations.

(A) Representative 3D light-sheet images of cleared whole FT from 1,2,5 months old MADM-wildtype mice $(n=3)$. The yellow box shows small clones, the magenta box shows expanded clones. Scale bar $=25 \mu \mathrm{m}$.

(B) Violin plot of clonal size distribution of wildtype clones at 1,2,5 months. Data pooled from 3 mice.

(C) Representative images of EdU labeling in expanded wildtype clones at 1,2,5 months. Scale bar $=20 \mu \mathrm{m}$.

(D) Representative images of EdU labeling in expanded mutant clones at 1,2,5 months. Scale bar $=20 \mu \mathrm{m}$.

(E) EdU+\% in each individual expanded wildtype and mutant clones at 1,2,5 months. Data pooled from three mice.

(F) Number of EdU+ cells in each individual expanded wildtype and mutant clones at 1,2,5 months. Data pooled from three mice.

(G) The average size of expanded wildtype clones and mutant clones from 1,2,5 months old mice. \pm SEM. t-test, ${ }^{* *}<0.01^{* * * *}<0.0001$. 


\section{Figure 4}

A

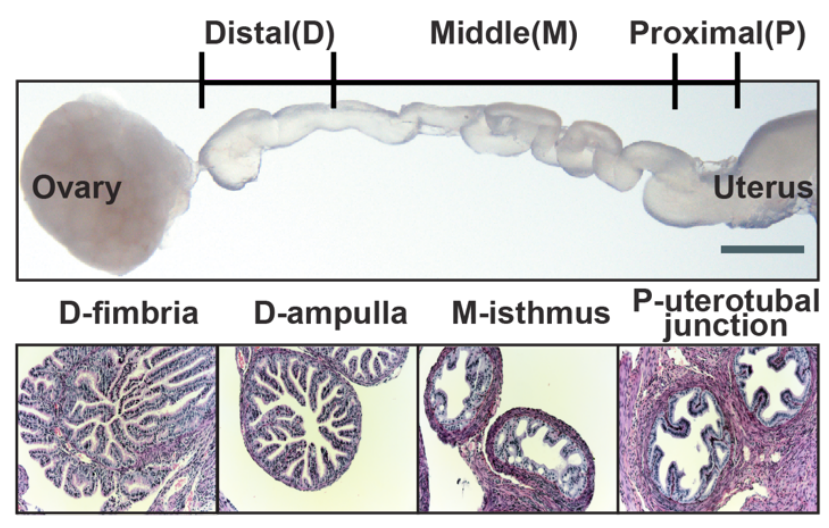

B
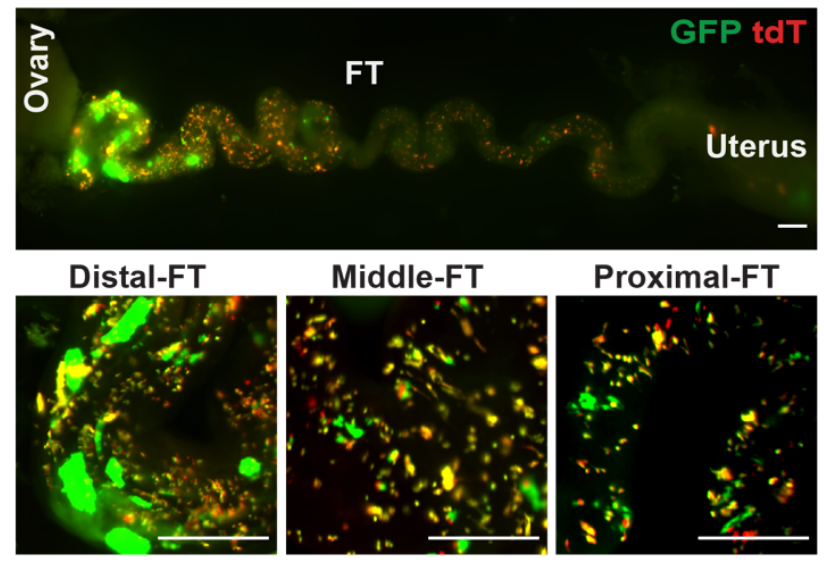

Proximal-FT
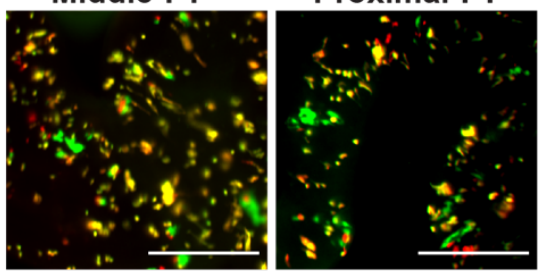

C

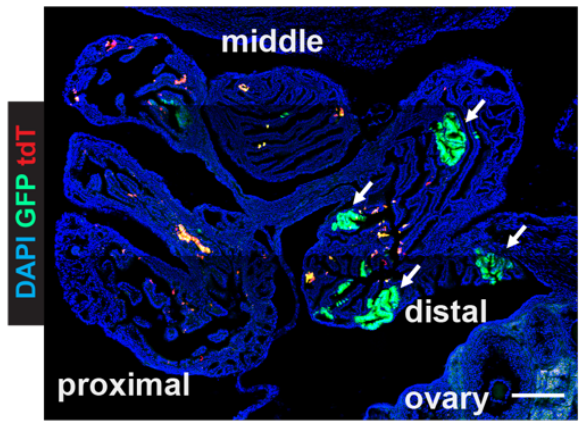

D

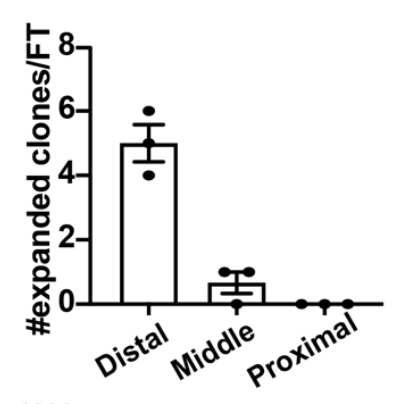

E

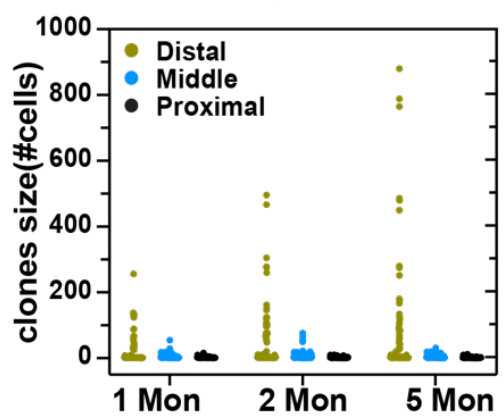


Fig. 4: The highly expanded mutant clones were spatially enriched in the distal FT.

(A) Upper panel: Whole-mount white-field image of a stretched FT from 2-month-old mutant mice which can be separated into three portions based on distance to uterus;

Lower panel: each FT region has distinct tubal morphology and inner folds shape. Scale bar $=500 \mu \mathrm{m}$.

(B) Upper panel: Whole-mount fluorescence image of the stretched FT; Lower panel: zoom into the distal, middle and proximal FT. Scale bar $=500 \mu \mathrm{m}$.

(C) Images of FT sections from 5-month-old mutant FTs $(n=3)$, showing that expanded mutant clones were mainly in the distal region. Scale bar=200 $\mu \mathrm{m}$.

(D) Quantification the number of expanded clones in distal(D), middle(M) and proximal $(\mathrm{P})$ regions. $( \pm \mathrm{SEM}, n=3)$.

(E) The size distribution of mutant clones in proximal, middle and distal FTs from 1,2,5months old MADM-mutant mice. Data pooled from four mice. 
bioRxiv preprint doi: https://doi.org/10.1101/2022.01.23.477434; this version posted January 24,2022 . The copyright holder for this preprint (which was not certified by peer review) is the author/funder. All rights reserved. No reuse allowed without permission.

\section{Figure 5}

A

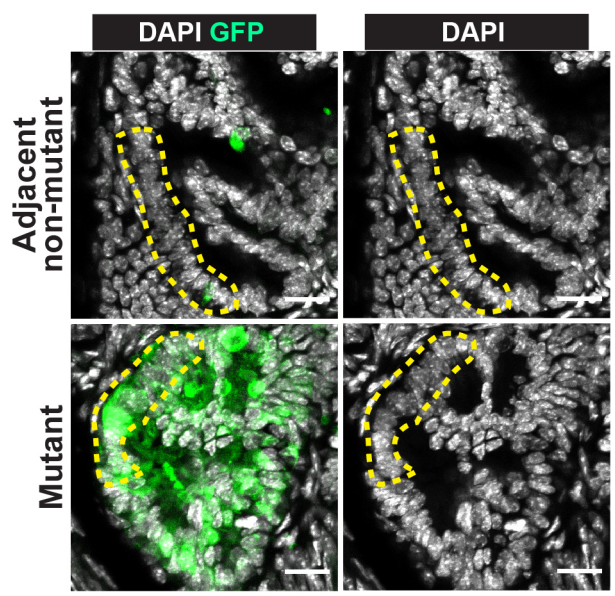

C

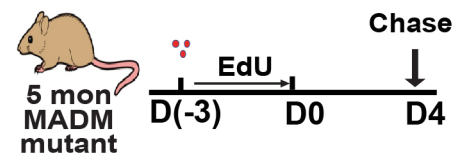

D
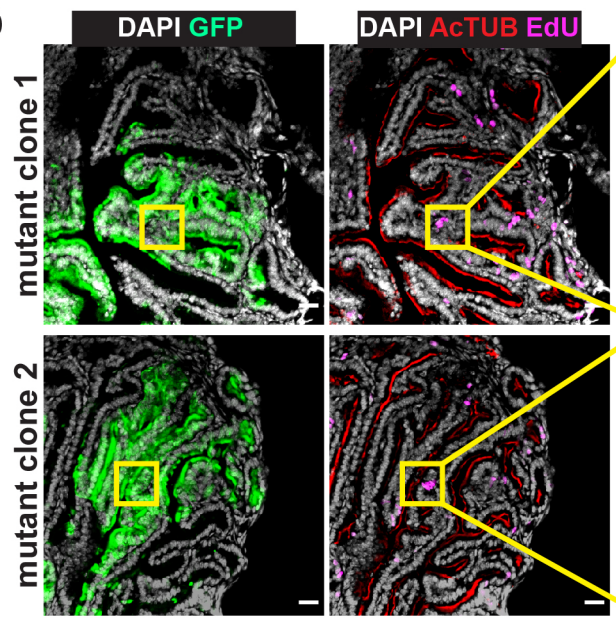

[1] EdU+cilia+

$\mathbf{F}$

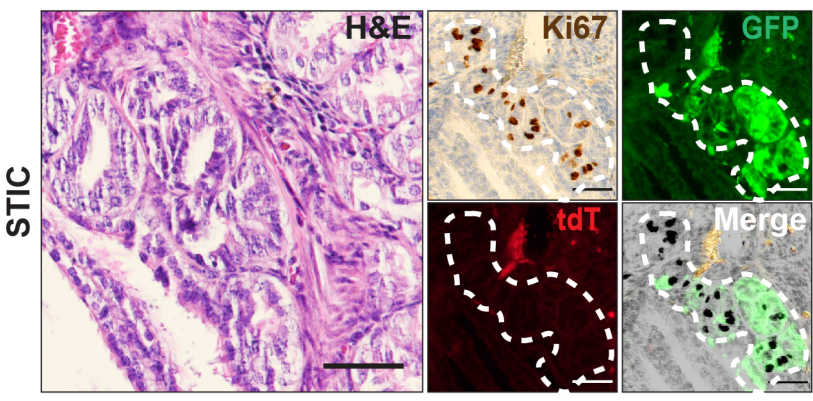

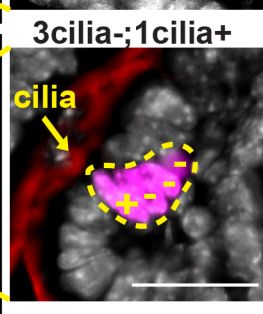

B
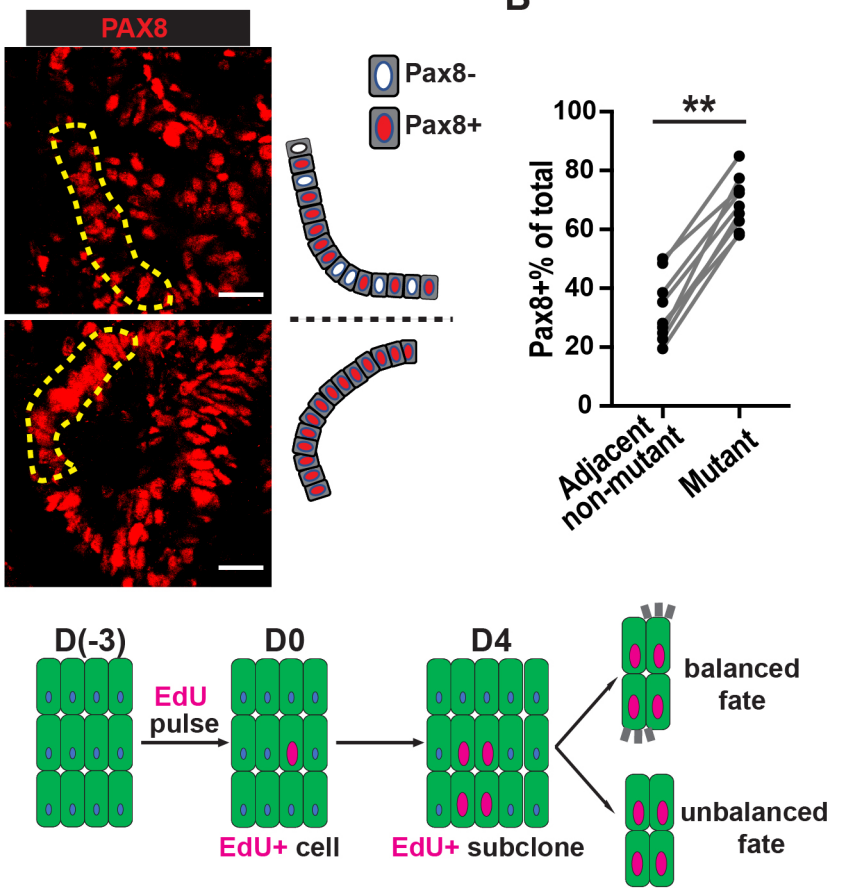
4cilia-
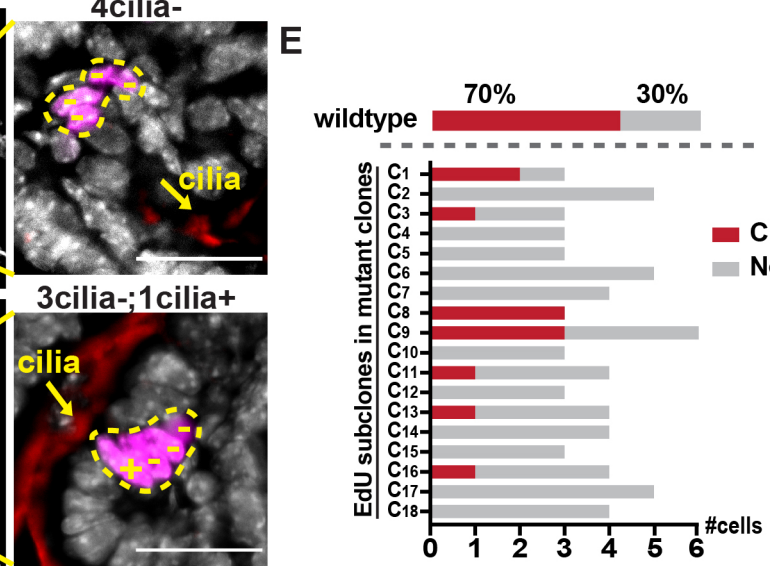

G

\begin{tabular}{cccc}
\hline \multirow{2}{*}{ Age(month) } & \multirow{2}{*}{ Total(n) } & \multicolumn{2}{c}{ Fallopian tube } \\
\cline { 2 - 4 } & & Normal & STIC \\
\hline 2 & 7 & 6 & $\mathbf{0}$ \\
3 & 5 & 5 & $\mathbf{0}$ \\
4 & 6 & 6 & $\mathbf{0}$ \\
5 & 9 & 9 & $\mathbf{0}$ \\
6 & 16 & 16 & $\mathbf{0}$ \\
8 & 7 & 6 & $\mathbf{1}$ \\
10 & 9 & 7 & $\mathbf{2}$ \\
12 & 5 & 3 & $\mathbf{2}$ \\
14 & 19 & $16^{*}$ & $\mathbf{1}$ \\
\hline Histopathological evaluation of FTs from
\end{tabular}

MADM-mutant mice. * in two mice, FTs were engulfed by uterine tumor 


\section{Figure 5: The progressive mutant clones showed increase propensity to produce} Pax8+ cells.

(A) Representative images of Pax8+ cell distribution in progressive mutant clones (lower panel), and in adjacent non-mutant regions (upper panel). The mutant clones showed continuous expansion of Pax8+ cells. Scale bar=20 $\mu \mathrm{m}$.

(B) The progressive mutant clones contained a higher portion of Pax $8+$ cells than adjacent non-mutant regions. Three mutant/non-mutant pairs were quantified from each of three mice in total. Wilcoxon-test, ${ }^{* *}<0.01$.

(C) The scheme to perform clonal tracing of proliferating cells within progressive clones through EdU labeling, and potential outcomes.

(D) Representative images show cellular composition of EdU subclones within progressive mutant clones. Upper panel shows a four-cell subclone with only nonciliated cells. Lower panel shows a mixture subclone with one ciliated cells (+) and three non-ciliated cells (-). Scale bar=25 $\mu \mathrm{m}$.

(E) The cellular composition of EdU+ spots (>2 cells) within the mutant clones. In wildtype distal FT, ciliated cells account for the majority, however, in the EdU subclones within mutant clones, non-ciliated cells account for the majority.

(F) Immunofluorescence staining showing overlap of STICs with GFP, not tdTomato. Scale bar $=50 \mu \mathrm{m}$.

(G) STICs frequency in the FTs from MADM-mutant mice of an age cohort. 


\section{Graphic Model.}

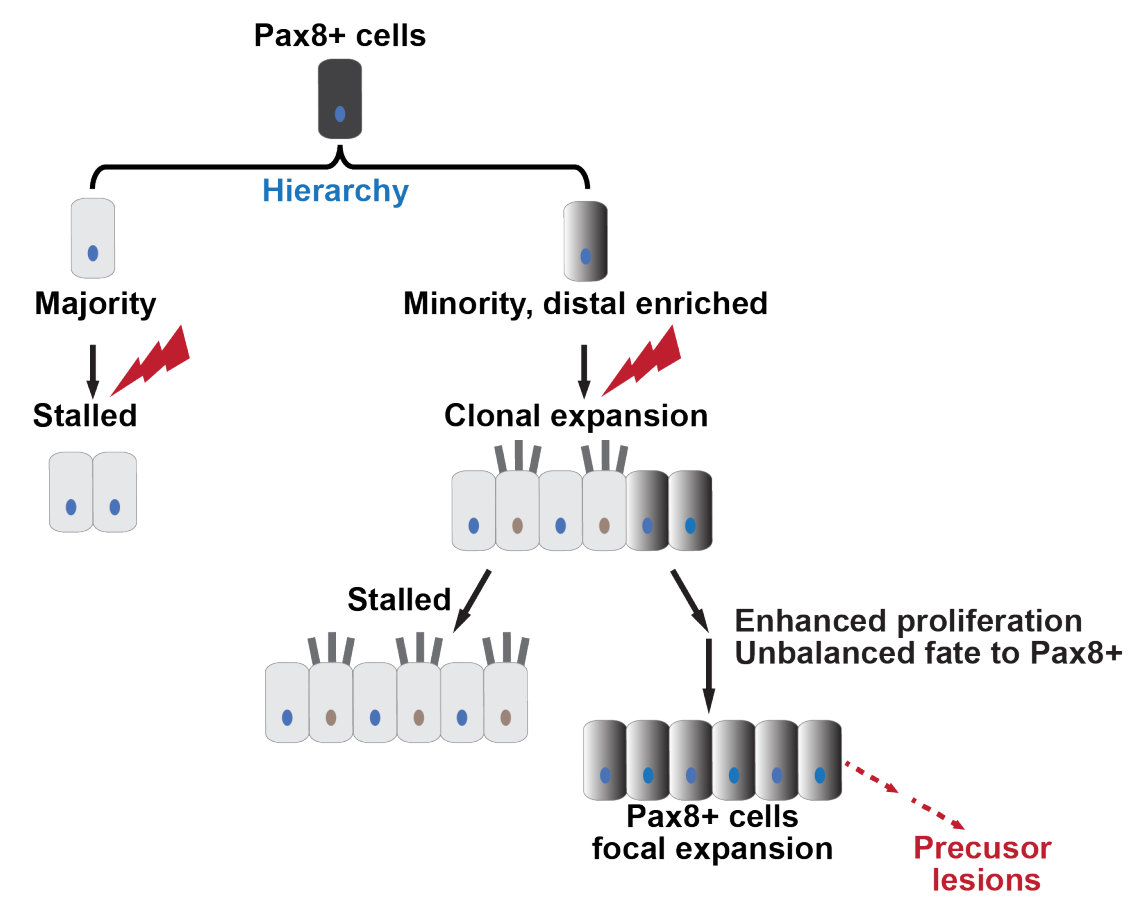

\section{Model delineating clonal dynamics of ovarian cancer-initiating cells in the fallopian tube}

The initiated FT Pax8+ cells adopt distinct clonal expansion fate shortly after being generated, with the majority stalled, a minority which are spatially enriched in the distal FT can quick expanded. The initially expanded mutant clones then experience a further divergence in their fate, either lose their progression or keep a long-term progression. The later one presents unbalanced differentiation and cause focal expansion of Pax8+ cells, which underlies cancer development. 
bioRxiv preprint doi: https://doi.org/10.1101/2022.01.23.477434; this version posted January 24,2022 . The copyright holder for this preprint (which was not certified by peer review) is the author/funder. All rights reserved. No reuse allowed without permission.

\section{Figure S1}

A

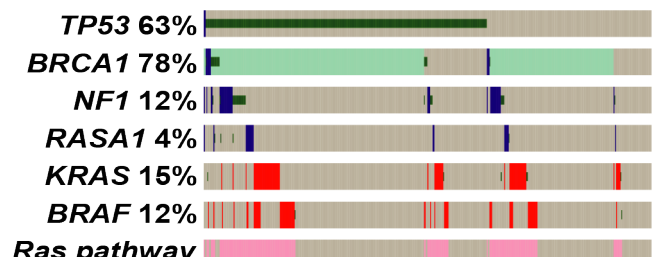

Ras pathway

$37 \%$

Genetic alterations IMissense |Shallow Deletion IDeep deletion IAmplifications IGain

C

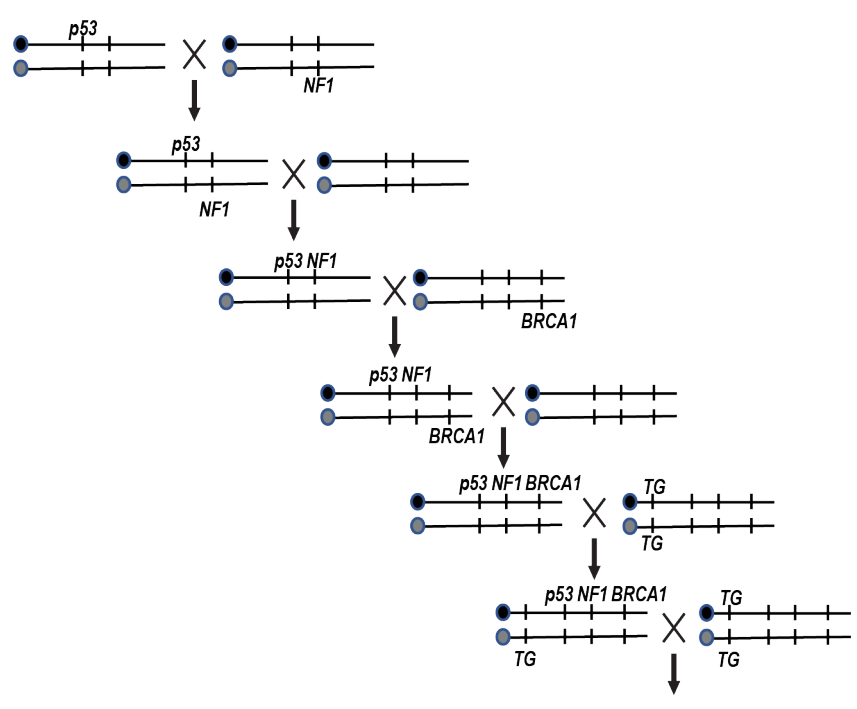

B

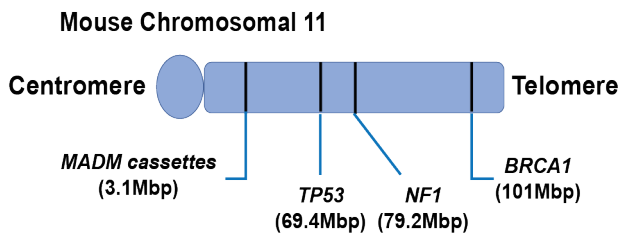

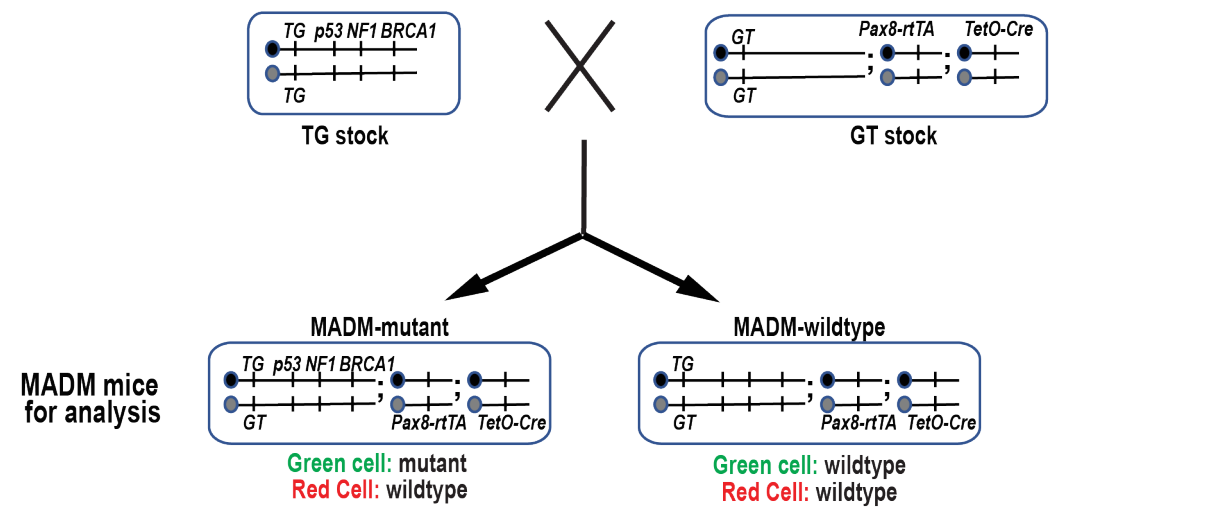


Fig. S1: Additional details for inducing initiated FT cells with MADM: mutation selection, chromosomal location of MADM cassettes and selected genes, and the breeding scheme.

(A) Assessment of HGSOC mutation spectrum with TCGA datasets.

(B) Chromosomal locations of MADM cassettes, TP53, BRCA1, NF1 on mouse chromosome 11. The physical locations were indicated

(C) The scheme to breed MADM-mutant mice and MADM-wildtype mice. We maintain two separate stocks and produce MADM mice through intercrossing the stocks. TP53, BRCA1 and NF1 mutations were incorporated into The TG stock, and the Cre transgenes (Pax8-rtTA, TetO-Cre) were incorporated into the GT stock. 


\section{Figure S2}

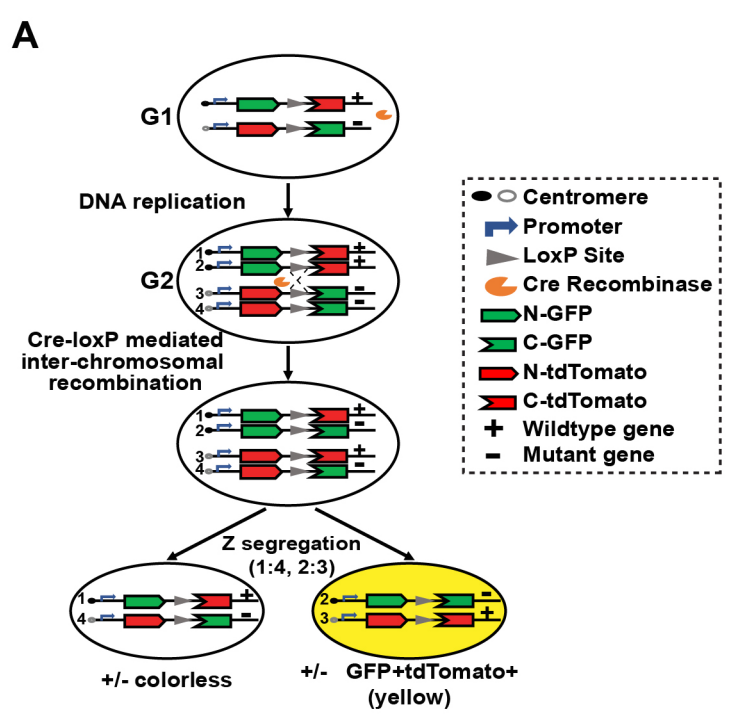

B

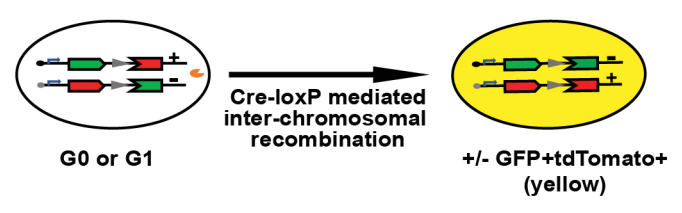

C
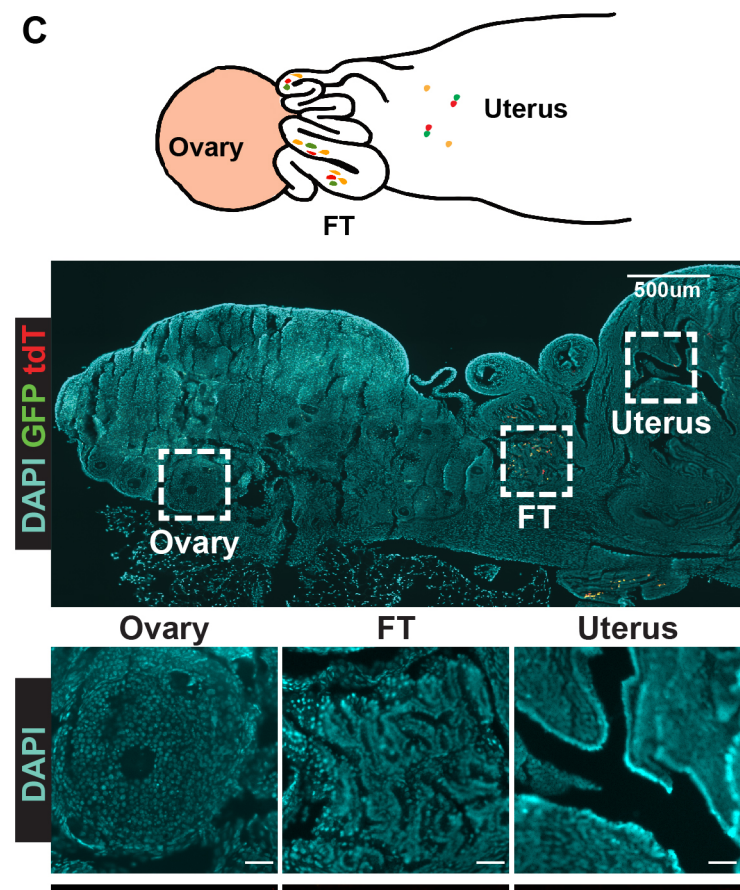

FT

Uterus
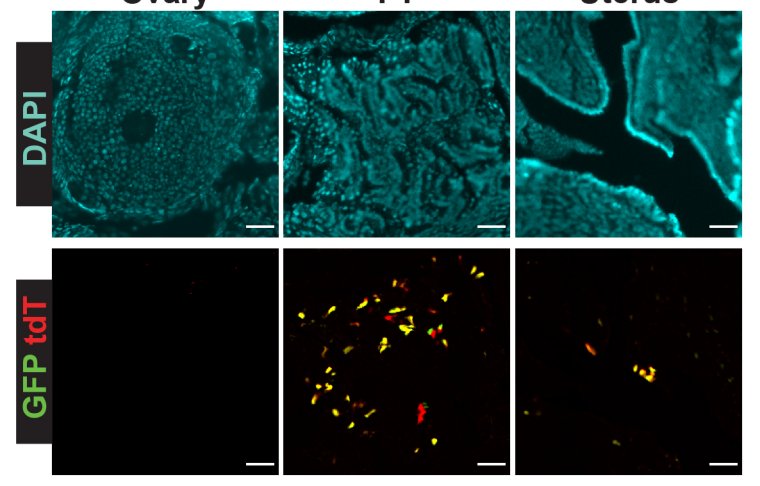

Fig. S2: Schematic mechanism of MADM to generate yellow cells without altering genotype, and MADM labeling specificity in the mouse reproductive tract.

(A) Cre-mediated inter-chromosomal mitotic recombination followed by $Z$ segregation generates either colorless or double-colored yellow cells without altering genotype.

(B) Cre-mediated inter-chromosomal recombination occurring in $\mathrm{G} 1$ or post-mitotic cells (G0) generates yellow cells without altering genotype.

(C) MADM labeled cells found in both the FT and the uterus, but not in the ovary. 


\section{Figure S3}

A

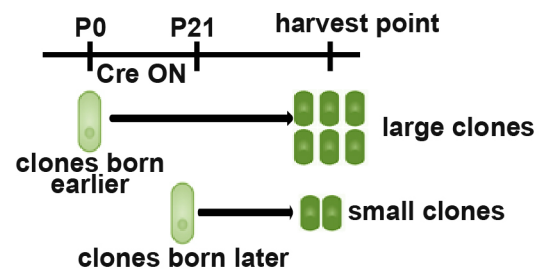

C

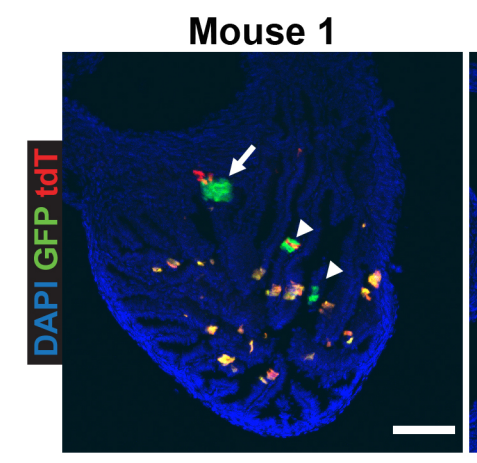

B

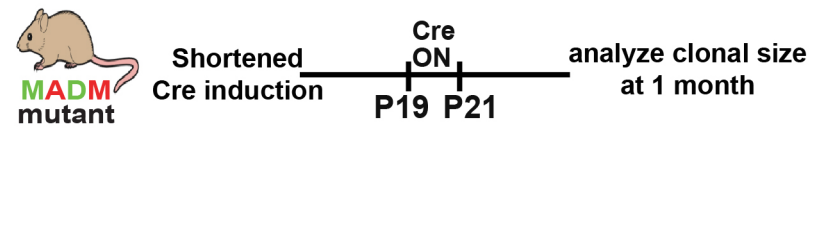

Mouse 2

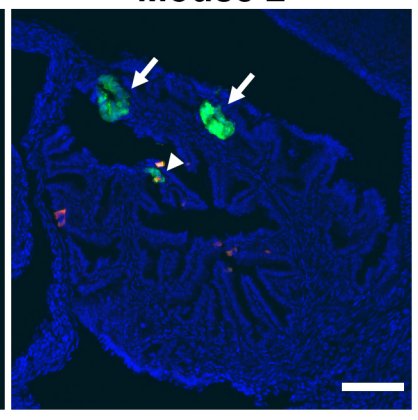

Mouse 3

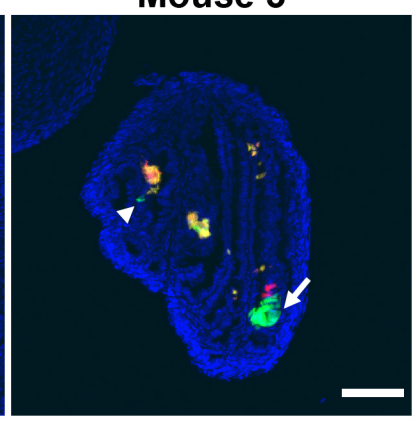

Fig. S3: Clonal size heterogeneity is not simply due to unsynchronized clonal age.

(A) The clonal age hypothesis: clones born earlier within the P0-21 range will show lager size at the harvest point, whereas clones born later will show smaller size.

(B) The scheme of DOX treatment to synchronize the clonal age of mutant cells.

(C) Representative images show heterogeneous size of MADM labeled clones in age synchronized mice $(n=3)$. Arrow shows expanded clones and arrowhead shows nonexpanded clones. Scale bar $=100 \mu \mathrm{m}$ 


\section{Figure S4}

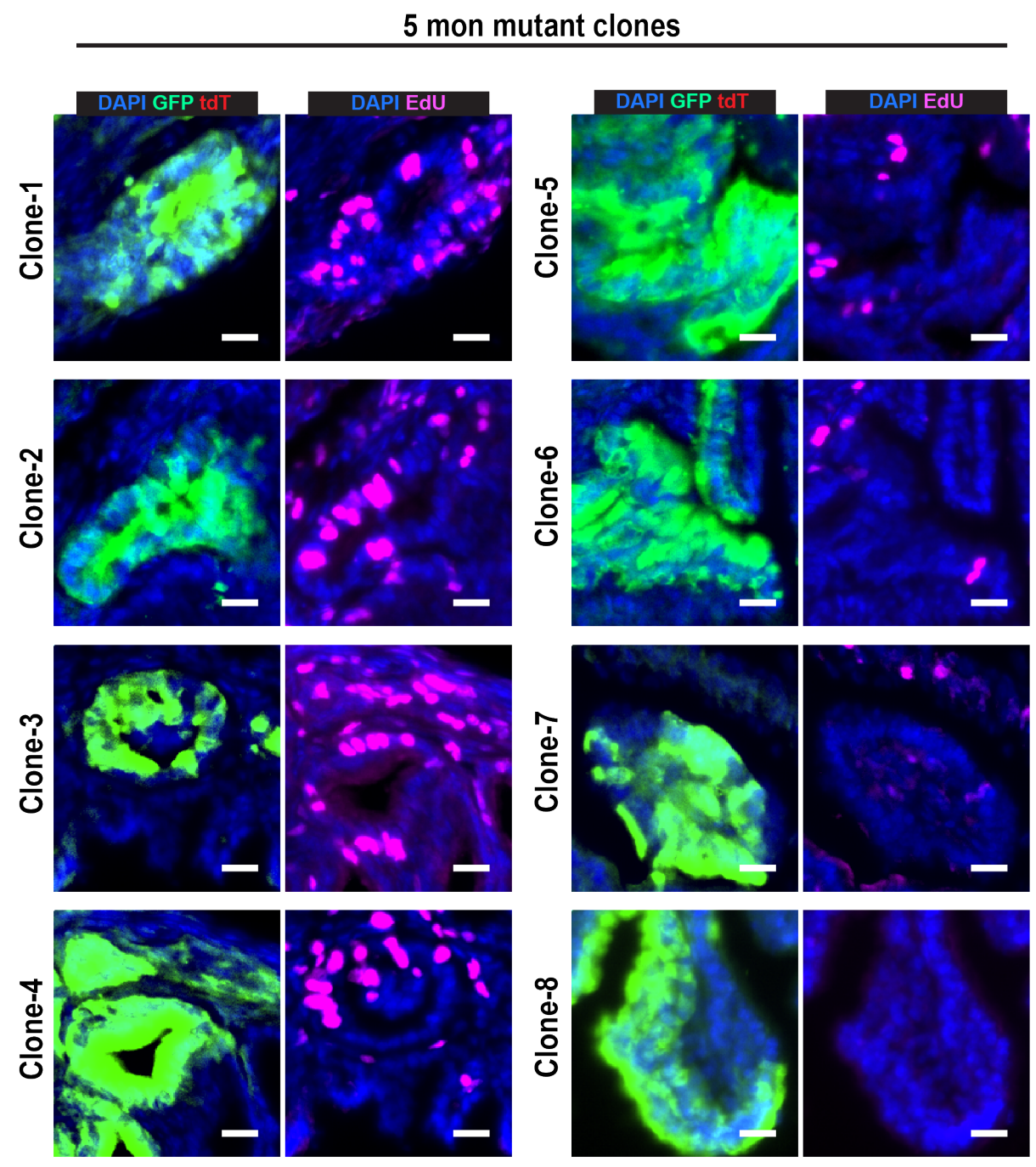

Fig. S4: More images of EdU labeling in expanded mutant clones at 5 months. Scale bar $=20 \mu \mathrm{m}$. 


\section{Figure S5}

A

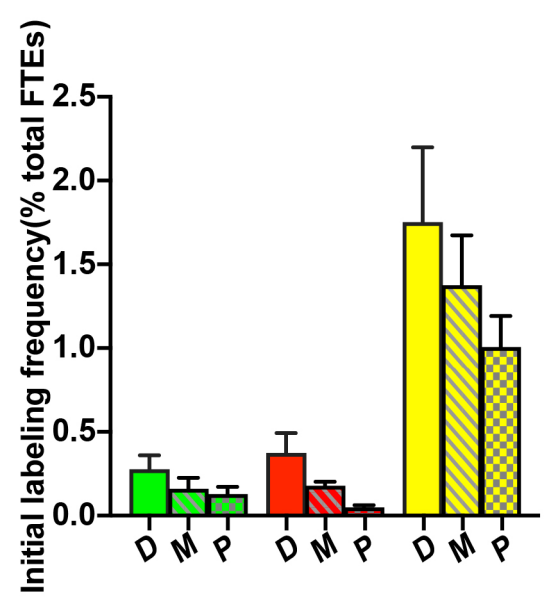

B
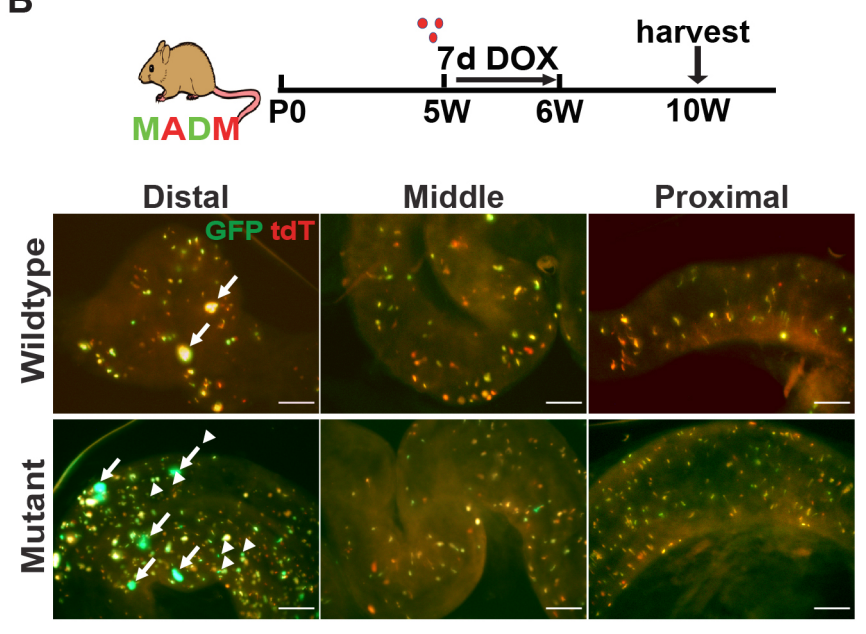

Fig. S5: MADM-labeling frequency in each FT segment and FTs with MADM clones induced post-developmentally.

(A) Initial MADM labeling frequency in distal(D), middle(M), and proximal(P) FT regions right after P0-21 Doxycycline administration $(n=3)$.

(B) Inducing MADM labeling in fully differentiated FTs: Doxycycline was administrated to MADM-wildtype/mutant mice between 5-6 weeks, then FTs were assessed at 10 weeks. Upper panel: wildtype FT; Lower panel: mutant FT. Expanded clones (arrow) and single cell clones were shown (arrowhead). Scale bar=200 $\mu \mathrm{m}$. 


\section{Figure S6}
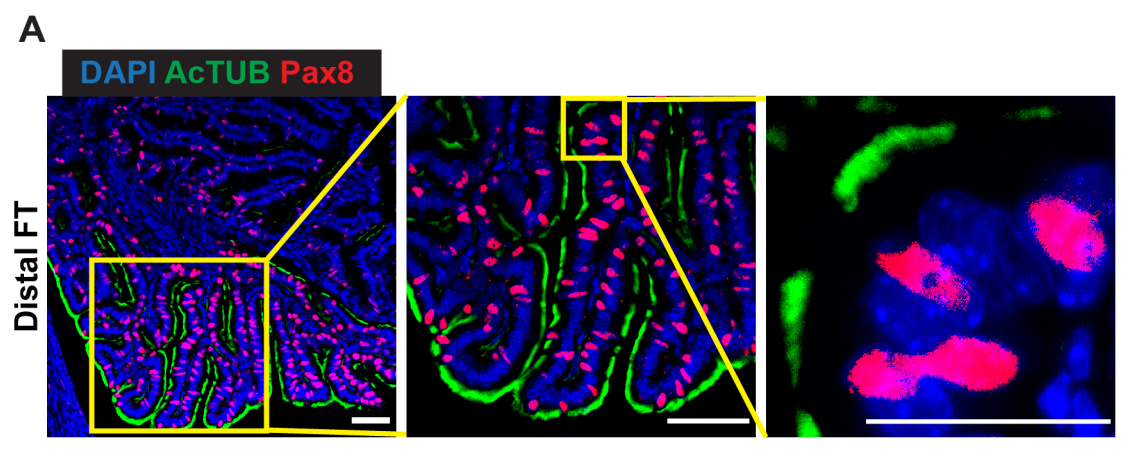

B

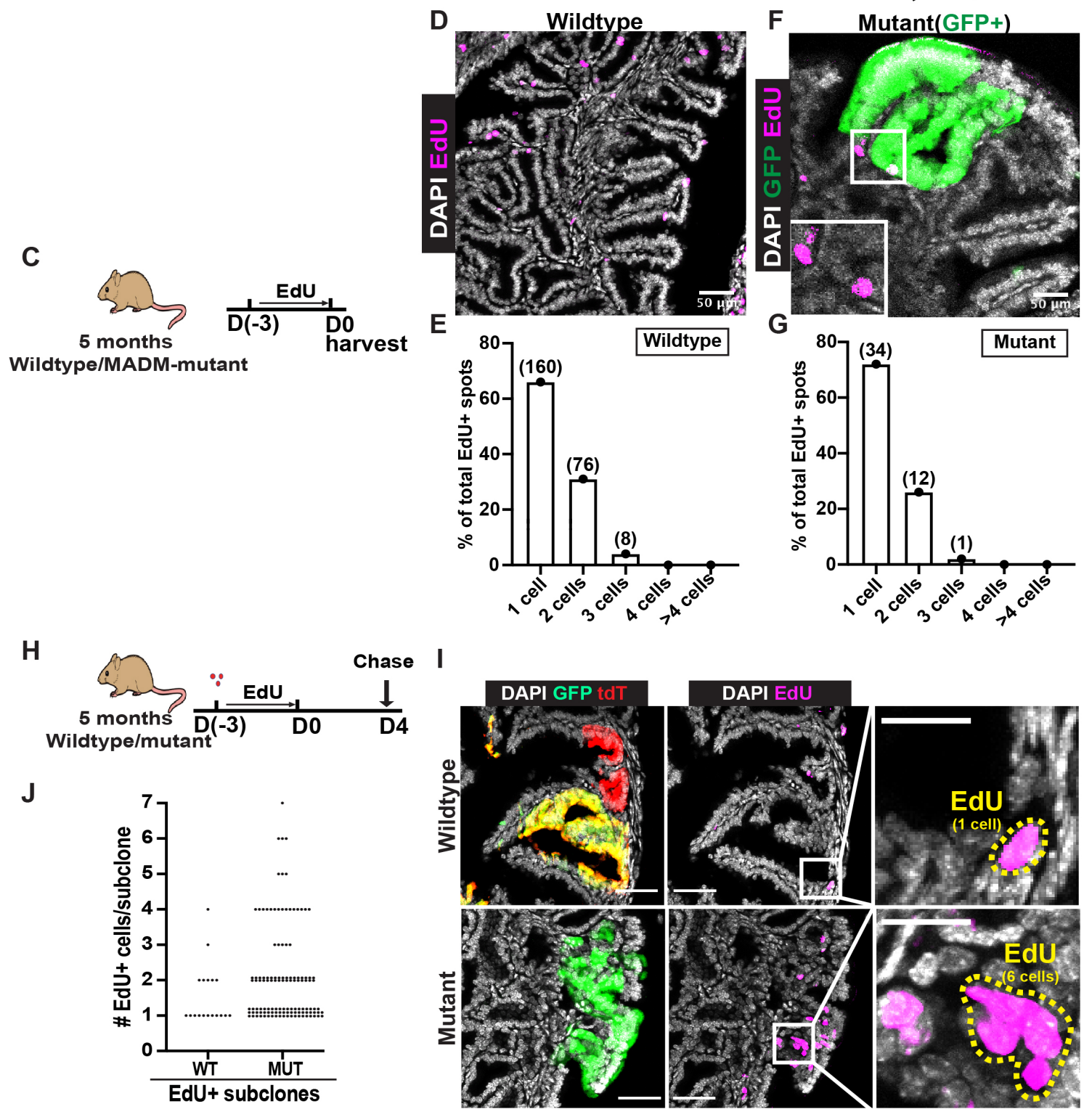


Fig. S6: The Pax8+ cell distribution pattern in wildtype distal FT, and 3-day EdU labeling marked cells at clonal density.

(A) Alternating Pax8+ and AcTUB+ cells in the distal FT from 5 months wildtype mice. Scale bar $=50 \mu \mathrm{m}$ for left and middle panels, scale bar=25 $\mu \mathrm{m}$ for right panel.

(B) Percentage of Pax8+ and Pax8- cells in the distal FT. ( \pm SEM, $n=3)$.

(C) Scheme for EdU treatment and analysis.

(D) EdU staining in wildtype FTs revealed that EdU labeled cells were scattered, and most EdU+ spots shown as one cell or two cells status. Scale bar=50 $\mu$ m. Representative image from 3 mice.

(E) The size distribution of EdU+ spots in FTs from 5 months old wildtype mice $(n=3)$. The total number of EdU spots $(n)$ is showed above the bar graph.

(F) EdU staining in mutant FTs revealed that EdU labeled cells were also scattered, and most EdU+ spots still shown as one cell or two cells. Scale bar=50 $\mu \mathrm{m}$. Representative image from 3 mice.

(G) The size distribution of all EdU+ spots in FTs from 5 months MADM-mutant mice $(n=3)$. The total number of EdU spots $(n)$ is shown above the bar graph.

(H) Scheme for EdU pulse-chase assay.

(I) Upper panel: EdU clone of one cell within expanded wildtype clones when chased at day4. Lower panel: EdU clone of six cells within progressive mutant clones. Scale bar $=20 \mu \mathrm{m}$.

(J) The size of each EdU clone within expanded wildtype clones and progressive mutant clones. Data pooled from three mice for each group. 